\title{
The effect of lattice vibrations on substitutional alloy thermodynamics
}

\author{
A. van de Walle and G. Ceder \\ Department of Materials Science and Engineering, Massachusetts Institute of Technology, \\ Cambridge, Massachusetts 02139
}

(Published 28 January 2002)

\begin{abstract}
A long-standing limitation of first-principles calculations of substitutional alloy phase diagrams is the difficulty in accounting for lattice vibrations. A survey of the theoretical and experimental literature seeking to quantify the effect of lattice vibrations on phase stability indicates that they can be significant. Typical vibrational entropy differences between phases are of the order of 0.1 to $0.2 k_{B}$ /atom, which is comparable to the typical values of configurational entropy differences in binary alloys (at most $0.693 k_{B}$ /atom). This article presents the basic formalism underlying ab initio phase diagram calculations, along with the generalization required to account for lattice vibrations. The authors review the various techniques allowing the theoretical calculation and the experimental determination of phonon dispersion curves and related thermodynamic quantities, such as vibrational entropy or free energy. A clear picture of the origin of vibrational entropy differences between phases in an alloy system is presented that goes beyond the traditional bond counting and volume change arguments. Vibrational entropy change can be attributed to the changes in chemical bond stiffness associated with the changes in bond length that take place during a phase transformation. This so-called "bond stiffness vs bond length" interpretation both summarizes the key phenomenon driving vibrational entropy changes and provides a practical tool to model them.
\end{abstract}

\section{CONTENTS}

I. Introduction

II. The Formalism of Alloy Theory
A. The cluster expansion formalism
B. The effect of lattice vibrations
C. Coarse graining of the partition function
D. Conclusion

III. Evidence of Vibrational Effects
A. Calculations and predicted effects on phase stability
B. Comparison with experiments
C. Conclusion

IV. The Origin of Vibrational Entropy Differences Between Phases
A. The "bond proportion" effect
B. The volume effect
C. The size mismatch effect

V. Computational Techniques
A. Lattice vibrations in the harmonic approximation
1. Theory
2. Force-constant determination
B. Anharmonicity
1. The quasiharmonic model
2. Simulation
C. Energy models
D. Convergence issues
1. Short-range force constant
2. Short-range effective cluster interactions

VI. Experimental Techniques

VII. Models of Lattice Vibrations
A. The "bond proportion" model
B. The Debye model
C. The Einstein model
D. The "bond stiffness vs bond length" approach

VIII. Conclusion

Acknowledgments

Appendix A: The Absence of Mass Effects in the High-

Temperature Limit

Appendix B: A Simple Model of Anharmonicity
Appendix C: Modeling the Disordered State Appendix D: The Einstein Model

Appendix E: Derivation of the Bond Proportion Model

Appendix F: Instability

References

\section{INTRODUCTION}

New materials having desirable properties are often discovered by alloying elements (or compounds) that possess some of the wanted qualities. As both computing power and the efficiency of computational methods are steadily increasing, it is becoming possible to investigate new alloys through computer simulations, before they have even been synthesized. However, before such a "virtual" material can make its way into the real world, it must pass the stringent test of thermodynamic stability. For this reason, the determination of alloy phase diagrams from first principles is among the most important steps required to build a "virtual laboratory" where materials could be designed from first principles without relying on experimental input.

The field of first-principles alloy theory, whose focus is the calculation of solid-state phase diagrams, has made substantial progress over the last two decades. Several excellent reviews on the topic exist (de Fontaine, 1994; Ducastelle, 1991; Zunger, 1994). As the thermodynamically stable form of most solid compounds is crystalline, alloy theory traditionally investigates the relative stability of phases characterized by a distinct ordering of atomic species on a given set of candidate lattices, allowing for small displacements away from the ideal lattice sites. Within that framework, it is now possible to predict relatively complex solid-state phase diagrams starting from the basic principles of quantum mechanics and statistical mechanics. Since no experimental input is required, these $a b$ initio calculations have been useful for clarifying the phase diagram of several new materials (Ceder et al., 1990; van der Ven et al., 1998). 
The accuracy of calculated phase diagrams is currently limited by two factors. First, one needs, as a starting point, the energy of the alloy in various atomic configurations and hence one is limited by the accuracy of the quantum-mechanical calculations used to obtain these energies. Typically, methods based on density-functional theory (DFT), such as the local-density approximation (LDA) or the generalized gradient approximation (GGA), are used.

A second shortcoming arises from the fact that, in order to reduce computational requirements, the sampling of the partition function to obtain the free energy is only done over a limited number of degrees of freedom. Typically, these include substitutional interchanges of atoms but no atomic vibrations. Attempts to either assess the validity of this approximation or to devise computationally efficient ways to account for lattice vibrations are currently the focus of intense research. This interest is fueled by the observation that phase diagrams obtained from first principles often incorrectly predict transition temperatures. It is hoped that lattice vibrations could account for some of the remaining discrepancies between theoretical calculations and experimental measurements.

Three main questions are addressed in this paper.

(1) Do lattice vibrations have a sufficiently important impact on phase stability that their thermodynamic effects need to be included in phase diagram calculations?

(2) What are the fundamental mechanisms that explain the relationship between the structure of a phase and its vibrational properties?

(3) How can the effect of lattice vibrations be modeled at a reasonable computational cost?

This paper is organized as follows. First, Sec. II presents the basic formalism that allows the calculation of phase diagrams, along with the generalization needed to account for lattice vibrations. A review of the theoretical and experimental literature seeking to quantify the impact of lattice vibrations on phase stability is then presented in Sec. III. The main mechanisms through which lattice vibrations influence phase stability are described in Sec. IV. Section V describes the methods used to calculate vibrational properties while Sec. VI presents the experimental techniques allowing their measurement. Finally, Sec. VII discusses the strengths and weaknesses of a variety of models of lattice vibrations.

\section{THE FORMALISM OF ALLOY THEORY}

Phase stability at constant temperature is determined by the free energy ${ }^{1} F$. The free energy can be expressed

\footnotetext{
${ }^{1}$ Strictly speaking, at constant pressure, the Gibbs free energy $G=F+P V$ should be used instead of the Helmoltz free energy $F$, but at atmospheric pressure, the $P V$ term is negligible for an alloy.
}

as a sum of a configurational contribution $F_{\text {config }}$ and vibrational contributions $F_{\text {vib }}$. The configurational contribution accounts for the fact that atoms can jump from one lattice site to another, while the vibrational contribution accounts for the vibrations of each atom around its equilibrium position. The first part of this section presents the traditional formalism used in alloy theory to determine the configurational contribution. The second part introduces the basic quantities that determine whether lattice vibrations have a significant effect on phase stability. The third part describes how the traditional formalism can be adapted when lattice vibrations do need to be accounted for.

\section{A. The cluster expansion formalism}

One of the goals of alloy theory is to determine the relative stability of phases characterized by a distinct ordering of atomic species on a given periodic array of sites. This array of sites, called the parent lattice, can be any crystallographic lattice augmented by any motif. Although the cluster expansion formalism apparently assumes a single parent lattice, alloy systems where the thermodynamically stable phases are based on multiple parent lattices can be handled by constructing a separate cluster expansion for each parent lattice.

A convenient representation of an alloy system is the Ising model. In the common case of a binary alloy system, the Ising model consists of assigning a spinlike occupation variable $\sigma_{i}$ to each site $i$ of the parent lattice, which takes the value -1 or +1 depending on the type of atom occupying the site. A particular arrangement of spins of the parent lattice is called a configuration and can be represented by a vector $\sigma$ containing the value of the occupation variable for each site in the parent lattice. Although this framework can be extended to arbitrary multicomponent alloys (Sanchez et al., 1984), we focus on the case of binary alloys, since all the studies we review consider binary alloys only.

When all the fluctuations in energy are assumed to arise solely from configurational changes, the Ising model is a natural way to represent an alloy. The thermodynamics of the system can then be summarized in a partition function of the form

$$
Z=\sum_{\sigma} \exp [-\beta E(\sigma)]
$$

where $\beta=1 /\left(k_{B} T\right)$, and $E(\sigma)$ is the energy when the alloy has configuration $\sigma$. It would be computationally intractable to compute the energy of every configuration from first principles. Fortunately, the configurational dependence of the energy can be parametrized in a compact form with the help of the so-called cluster expansion (Sanchez et al., 1984). The cluster expansion is a generalization of the well-known Ising Hamiltonian. The energy (per atom) is represented as a polynomial in the occupation variables: 


$$
\frac{E(\sigma)}{N}=\sum_{\alpha} m_{\alpha} J_{\alpha}\left(\prod_{i \in \alpha^{\prime}} \sigma_{i}\right),
$$

where $\alpha$ is a cluster (a set of sites $i$ ). The sum is taken over all clusters $\alpha$ that are not equivalent by a symmetry operation of the space group of the parent lattice, while the average is taken over all clusters $\alpha^{\prime}$ that are equivalent to $\alpha$ by symmetry. The coefficients $J_{\alpha}$ in this expansion embody the information regarding the energetics of the alloy and are called the effective cluster interaction (ECI). The multiplicities $m_{\alpha}$ indicate the number of clusters that are equivalent by symmetry to $\alpha$ (divided by the number of lattice sites). ${ }^{2}$

It can be shown that when all clusters $\alpha$ are considered in the sum, the cluster expansion is able to represent any function $E(\sigma)$ of configuration $\sigma$ by an appropriate selection of the values of $J_{\alpha}$. However, the real advantage of the cluster expansion is that, in practice, it is found to converge rapidly. A sufficient accuracy for phase diagram calculations can be achieved by keeping only clusters $\alpha$ that are relatively compact (e.g., shortrange pairs or small triplets). The unknown parameters of the cluster expansion (the effective cluster interaction) can then be determined by fitting them to the energy of a relatively small number of configurations obtained, for instance, through first-principles computations. The cluster expansion thus presents an extremely concise and practical way to model the configurational dependence of an alloy's energy.

How many ECI and structures are needed in practice? A typical well-converged cluster expansion of the energy of an alloy consists of about 20-30 effective cluster interactions and necessitates the calculation of the energy of around 40-50 ordered structures (see, for instance, Garbulksy and Ceder, 1995; Ozoliņš et al., 1998a; van der Ven et al., 1998). A faithful modeling of the qualitative features of the phase diagram (correct stable phases and topology) typically requires far fewer effective cluster interactions (as little as one pair interaction) and correspondingly less structures, as illustrated by the numerous examples given in de Fontaine (1994) and Ducastelle (1991). In general multicomponent systems, the number of effective cluster interactions and ordered structures required to achieve a given precision unfortunately grows rapidly with the number of species (Sanchez et al., 1984). For instance, in ternaries, each pair interaction is characterized by three interaction parameters instead of only one in the binary case. For this reason, very few first-principle calculations of ternary phase diagrams have been attempted [see McCormack and de Fontaine (1996) for a recent example, or de Fontaine (1994) for a survey].

Although the cluster expansion usually allows a very compact representation of the energetics of an alloy sys-

\footnotetext{
${ }^{2}$ Both the number of clusters and the number of sites are infinite but their finite ratio can be obtained by ignoring all but one periodic repetitions of the clusters (or the atoms) by the translational symmetry operations of the lattice.
}

tem, there are two situations where a standard cluster expansion is known to converge slowly. Systems where long-range elastic interactions are important due to a large atomic size mismatch between the alloyed species may require that elastic interactions be explicitly accounted for through the use of a so-called reciprocal space cluster expansion (Ozoliņš et al., 1998a, 1998b; Zunger, 1994). Another situation, as recently identified by Johnson et al. (2000), is when the electronic structure of the system exhibits a very strong configurational dependence due to symmetry-breaking effects.

In the cases where a short-range cluster expansion does provide a sufficient accuracy, the process of calculating the phase diagram of an alloy system can be summarized as follows. First, the energy of the alloy in a relatively small number of configurations is calculated, for instance through first-principles computations. Second, the calculated energies are used to fit the unknown coefficients of the cluster expansion (the effective cluster interaction $J_{\alpha}$ ). Finally, with the help of this compact representation, the energy of a large number of configurations is sampled, in order to determine the phase boundaries. This latter step can be accomplished with either the cluster variation method (CVM) (Ducastelle, 1991; Kikuchi, 1951), the low-temperature expansion (LTE) (Kohan et al., 1998), or Monte Carlo simulations (Binder and Heermann, 1988).

\section{B. The effect of lattice vibrations}

The previous section described the framework allowing the calculation of phase diagrams under the assumption that the thermodynamics of the alloy is determined solely by configurational excitations. Accounting for vibrational excitations introduces corrections to this simplified treatment. This section presents the basic quantities that enable an estimation of the magnitude of the effect of lattice vibration on alloy thermodynamics. To understand the effect of lattice vibrations on phase stability, it is instructive to decompose the configurational ("config") and vibrational ("vib") parts of the free energy $F^{\alpha}$ of a phase $\alpha$ into an energetic contribution $E$ and an entropic contribution $S$ :

$$
F^{\alpha}=E_{\text {config }}^{\alpha}-T S_{\text {config }}^{\alpha}+E_{\mathrm{vib}}^{\alpha}-T S_{\text {vib }}^{\alpha} .
$$

We take the convention that $E_{\text {config }}^{\alpha}$ is the energy of the alloy system when all atoms are frozen at their average position at a given temperature. In the approximation of harmonic lattice vibrations and in the limit of high temperature, the vibrational energy $E_{\text {vib }}^{\alpha}$ is simply determined by the equipartition theorem and is independent of the phase $\alpha$ considered. Hence as long as these approximations are appropriate, lattice vibrations are mainly expected to influence phase stability through their entropic contribution $S_{\text {vib }}^{\alpha}$.

Intuitively, the vibrational entropy $S_{\text {vib }}^{\alpha}$ is a measure of the average stiffness of an alloy, as can be best illustrated by considering a simple system made of a large number of identical harmonic oscillators. The softer the oscilla- 
tors are, the larger their oscillation amplitude can be, for a fixed average energy per oscillator. Hence the system samples a larger number of states and the entropy of the system increases. In summary, the softer the alloy, the larger the vibrational entropy.

A phase with a large vibrational entropy is stabilized relative to other phases, since a larger vibrational entropy results in a lower free energy, as seen by Eq. (3). From a statistical-mechanics point of view, this fact can be understood by observing that a phase that encloses more states in phase space is more likely to be visited, as the system undergoes microscopic transitions, and therefore exhibits an increased stability.

The central role of vibrational entropy can be further appreciated by considering the effect of vibrations on a phase transition between two phases $\alpha$ and $\beta$ which differ only by their average configuration (e.g., an orderdisorder transition). If the vibrational entropy difference between the two phases is $\Delta S_{\mathrm{vib}}^{\alpha \rightarrow \beta}$, the transition temperature obtained with both configurational and vibrational contributions ( $T_{\text {config }+ \text { vib }}^{\alpha \rightarrow \beta}$ ) is related to the transition temperature obtained with configurational effects only $\left(T_{\text {config }}^{\alpha \rightarrow \beta}\right)$ by

$$
T_{\text {config vib }}^{\alpha \rightarrow \beta} \approx T_{\text {config }}^{\alpha \rightarrow \beta}\left(1+\frac{\Delta S_{\text {vib }}^{\alpha \rightarrow \beta}}{\Delta S_{\text {config }}^{\alpha \rightarrow \beta}}\right)^{-1},
$$

where $\Delta S_{\text {config }}^{\alpha \rightarrow \beta}$ is the change in configurational entropy upon phase transformation (Garbulsky and Ceder, 1994). This result is exact in the limit of small vibrational effects, high temperature, and harmonic vibrations. A correction to this result that accounts for anharmonicity can be found in Ozolinš et al. (1998c). Equation (4) indicates that the quantity determining the magnitude of the effect of lattice vibration on phase stability is the ratio of the vibrational entropy difference to the configurational entropy difference. For this reason, most investigations aimed at assessing the importance of lattice vibrations focus on estimating vibrational entropy differences between phases. Since the configurational entropy (per atom) $S_{\text {config }}$ for a binary alloy at concentration $c$ is bracketed by

$$
\begin{aligned}
0 \leqslant S_{\text {config }} & \leqslant-k_{B}[c \ln c+(1-c) \ln (1-c)] \\
& \leqslant k_{B} \ln 2 \approx 0.693 k_{B},
\end{aligned}
$$

Eqs. (4) and (5) provide us with an absolute scale to gauge the importance of vibrations. As we will see, typical vibrational entropy differences are of the order of $0.2 k_{B}$, indicating that corrections of the order of $30 \%$ to the transition temperature may not be uncommon.

While it is clear that vibrational excitations introduce quantitative corrections to the simple picture of alloy thermodynamics based on configurational excitations only, more profound effects of a qualitative nature are also possible. Vibrational effects may lead to deviations from the traditional belief that, at high enough temperature, all short-range order in a disordered material disappears (Garbulsky and Ceder, 1994; Miller, 1989).
While a fully disordered state clearly maximizes configurational entropy $S_{\text {config }}$, it is not clear that the total entropy $S_{\text {config }}+S_{\text {vib }}$ is necessarily maximized in the state of maximum configurational disorder. The presence of short-range order may increase the total entropy, relative to a fully disordered alloy, through an increase of the vibrational entropy. Vibrational entropy somewhat challenges our intuition, which is largely derived from tacitly assuming that configurational disorder is all disorder. It is even conceivable that vibrational entropy could induce a transition from a disordered to an ordered phase with increasing temperature, if the vibrational entropy difference between the ordered and disordered phases is larger and opposite to the configurational entropy difference. While this phenomenon has, so far, not been observed in metallic alloys, presumably because of the large configurational entropy associated with disordering, it does occur in molecular systems, such as in diblock copolymer melts, where the configurational entropy (per monomer) is small (Russell et al., 1994).

\section{Coarse graining of the partition function}

The cluster expansion formalism presented in Sec. II.A appears to focus solely on configurational excitations. This section shows that, in fact, nonconfigurational sources of energy fluctuations can naturally be taken into account within the cluster expansion framework through a process called "coarse graining" of the partition function ${ }^{3}$ (Ceder, 1991, 1993). This procedure also clarifies the nature of the physical states that are represented by a configuration of the Ising model.

All the thermodynamic information of a system is contained in its partition function:

$$
Z=\sum_{i} \exp \left[-\beta E_{i}\right]
$$

where $E_{i}$ is the energy of the system in state $i$. In the case of a crystalline alloy system, the sum over all possible states of the system can be conveniently factored as follows:

$$
Z=\sum_{L} \sum_{\sigma \in L} \sum_{v \in \sigma} \sum_{e \in v} \exp [-\beta E(L, \sigma, v, e)],
$$

where

- $L$ is a so-called parent lattice: it is a set of sites where atoms can sit. In principle, the sum would be taken over any Bravais lattice augmented by any motif;

- $\sigma$ is a configuration on the parent lattice: It specifies which type of atom sits on each lattice site;

\footnotetext{
${ }^{3}$ This coarse graining process is, of course, unrelated to any processing aimed at increasing the grain size in a polycrystalline material.
} 
- $v$ denotes the displacement of each atom away from its ideal lattice site;

- $e$ is a particular electronic state (both the spatial wave function and spin state) when the nuclei are constrained to be in a state described by $v$;

- $E(L, \sigma, v, e)$ is the energy of the alloy in a state characterized by $L, \sigma, v$, and $e$.

Each summation is taken over the states that are included in the set of states defined by the "coarser" levels in the hierarchy of states. For instance, the sum over displacements $v$ includes all displacements such that the atoms remain close to the undistorted configuration $\sigma$ on lattice $L$.

While Eq. (7) is in principle exact, practical firstprinciples calculations of phase diagrams typically rely on various simplifying assumptions. The sum over electronic states is often reduced to a single term, namely, the electronic ground state. The validity of this approximation can be assessed by ensuring that different structures have a similar electronic density of states in the vicinity of the Fermi level. ${ }^{4}$ If needed, the contribution of electronic entropy is, at least in its one-electron approximation, relatively simple to include without prohibitive computational requirements (Wolverton and Zunger, 1995).

A simplifying assumption that is much more difficult to relax is the reduction of the sum over displacements $v$ to a single term. This simplification has been extensively used in alloy theory, because calculating the summation over $v$ involves intensive calculations. The particular displacement representing a given configuration $\sigma$ is typically chosen to be a local minimum in energy that is close to the undistorted ideal structure where atoms lie exactly at their ideal lattice sites. Usually, this state is found by placing the atoms at their ideal lattice positions and relaxing the system until a local minimum of the energy is obtained. In this fashion, the state chosen is the most probable one in the neighborhood of phase space associated with configuration $\sigma$. In this approximation, the partition function takes the form of an Ising model partition function:

$$
Z=\sum_{L} \sum_{\sigma \in L} \exp \left[-\beta E^{*}(L, \sigma)\right]
$$

with $E^{*}(L, \sigma)=\min _{v, e}\{E(L, \sigma, v, e)\}$.

It turns out that the same statistical-mechanics techniques developed in the context of the Ising model can also be used in the more general setting where atoms are allowed to vibrate (and where electrons are allowed to be excited above their ground state). All that is needed is to replace the energy $E^{*}(L, \sigma)$ by the constrained free energy $F(L, \sigma, T)$, defined as

\footnotetext{
${ }^{4}$ Unless strong electron correlation effects are present, such as charge ordering or metal-insulator transitions (Imada et al., 1998), the electronic free energy can be calculated from the single-electron density of states (DOS) in the neighborhood of the Fermi level.
}

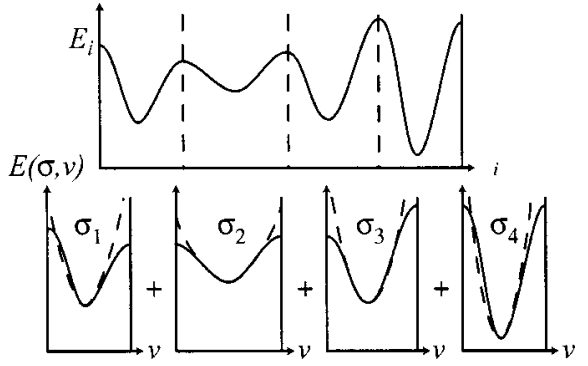

FIG. 1. The coarse graining approach. The global energy surface of an alloy system, which gives the energy $E_{i}$ of each state $i$, can be partitioned into a set of local energy surfaces, each of which is associated with a distinct configuration $\sigma_{k}$ of an Ising model. Within each configuration $\sigma_{k}$, the local energy surface gives the energy as a function of the atomic displacements $v$ (or any other nonconfigurational degrees of freedom). The thermodynamics of the system thus reduces to the one of a traditional Ising model, where the "energy" of each configuration now becomes the free energy associated with the corresponding local energy surface. If the local energy surfaces can be considered quadratic, their associated free energy can be determined by solving a Born-von Kármán phonon problem.

$F(L, \sigma, T)=-k_{B} T \ln \left(\sum_{v \in \sigma} \sum_{e \in v} \exp [-\beta E(L, \sigma, v, e)]\right)$.

In other words, it is the free energy of the alloy, when its state in phase space is constrained to remain in the neighborhood of the ideal configuration $\sigma$. This process, called the coarse graining of the partition function, is naturally interpreted as integrating out the "fast" degrees of freedom (e.g., vibrations) before considering "slower" ones (e.g., configurational changes) (Ceder, 1993). This process is illustrated in Fig. 1. The quantity to be represented by a cluster expansion is now the constrained free energy $F(L, \sigma, T)$. The only minor complication is that the effective cluster interactions become temperature dependent.

There is some level of arbitrariness in the precise definition of the set of displacement $v$ over which the summation is taken in Eq. (9). However, in the common case where there is a local energy minimum in the neighborhood of $\sigma$ and where the system spends most of its time visiting a neighborhood that can be approximated by a harmonic potential well, the set of displacements over which the summation is taken has little effect on the calculated thermodynamic properties. Under the above assumptions, calculating the partition function of a constrained harmonic system and a harmonic system that allows infinite displacements gives essentially the same result:

$$
\begin{aligned}
\sum_{v \in \sigma} & \exp [-\beta E(L, \sigma, v, T)] \\
& \approx \sum_{v \in \sigma} \exp \left[-\beta E_{H}(L, \sigma, v, T)\right] \\
& \approx \sum_{\text {all } v} \exp \left[-\beta E_{H}(L, \sigma, v, T)\right],
\end{aligned}
$$


where $E(L, \sigma, v, T)=-k_{B} T \ln \Sigma_{e \in v} \exp [-\beta E(L, \sigma, v, e)]$ and $E_{H}(L, \sigma, v, T)$ denotes a harmonic approximation to $E(L, \sigma, v, T)$. In this framework, all that is needed to account for lattice vibrations is the determination of the free energy of a harmonic solid in the neighborhood of any configurations $\sigma$. (Appendix $\mathrm{F}$ discusses the case where the above assumptions are violated, that is, when no local minimum exists in the phase space neighborhood of $\sigma$.)

The problem of calculating $F(L, \sigma, T)$ for a set of configurations $\sigma$ is much more demanding than calculating the energy $E^{*}(L, \sigma)$ for a set of $\sigma$. Devising an efficient way to calculate $F(L, \sigma, T)$ is the fundamental problem that needs to be resolved in order to include vibrational effects in phase diagram calculations.

\section{Conclusion}

After presenting the basic framework enabling the calculation of the configurational free energy, this section has presented two important aspects of the thermodynamics of lattice vibrations.

(1) Vibrational entropy differences between phases introduce corrections to transition temperatures calculated with only configurational entropy.

(2) The basic alloy theory framework can be adapted to account for lattice vibrations by replacing the energy $E^{*}(L, \sigma)$ associated with each configuration $\sigma$ by the free energy $F(L, \sigma, T)$ of a system constrained to remain in the phase space neighborhood of the ideal configuration $\sigma$.

\section{EVIDENCE OF VIBRATIONAL EFFECTS}

In light of the large computational requirements associated with the inclusion of lattice vibrations, is it important to ensure that such an endeavor is worth the effort. This section reviews the experimental and theoretical evidence that supports the view that vibrational effects are important in the context of phase diagram calculations. There exists a large literature aimed at determining the vibrational properties of solids [see, for instance, Born and Huang (1956), Maradudin et al. (1971), Ashcroft and Mermin (1976)]. Here, the focus is on investigations directly related to the determination of vibrational entropy (or free-energy) differences between phases which differ solely by the ordering of the chemical species on an otherwise identical parent lattice.

This relatively narrow choice is driven by two observations. First, while there have been numerous investigations of the absolute vibrational properties of solids, the more difficult issue that needs to be addressed in the context of phase stability is the determination of accurate differences in vibrational properties. Second, it has already been established that many structural phase transformations (e.g., from fcc to bcc) are driven by lattice vibrations (Grimvall and Ebbsjo, 1975; Petry et al., 1991). This fact does not pose major difficulties for the purpose of phase diagram calculations: one can easily compute the vibrational properties of a few lattice types. The real difficulty is to calculate the vibrational entropy of many configurations on each of these lattices, a task which is only needed if vibrational properties differ substantially across distinct configurations on an identical parent lattice.

The presentation will be mainly chronological, although deviations from that intention will be made for the sake of clarity. We leave a more precise description of the methods used for subsequent sections, focusing here on the results obtained. The key theoretical and experimental results are summarized in Tables I-III respectively.

\section{A. Calculations and predicted effects on phase stability}

The idea that the state of order of an alloy could be coupled with its lattice dynamics is not new. During the 1960s, as the foundations of alloy thermodynamics were being established, the question of the effect of lattice vibrations was already being raised. Studies on the order-disorder transition of $\beta$-brass (Booth and Rowlinson, 1955; Wojtowicz and Kirkwood, 1960), for instance, have indicated that lattice vibrations are crucial to accurately model the magnitude of the experimentally observed discontinuity in heat capacity at the phase transition, which determines the change in vibrational entropy upon disordering.

After these initial investigations, increasingly accurate models for the coupling between lattice vibration and the state of order of an alloy were then developed (Bakker, 1982a, 1982b; Bakker and Tuijn, 1986; Garbulksy and Ceder, 1994; Matthew et al., 1983; Moraitis and Gautier, 1977; Tuijn and Bakker, 1989). These models generally involved unknown parameters that need to be estimated from available experimental thermodynamic data. A recurring theme among these studies is the idea that, for sensible choices of the stiffness of the springs connecting the atoms, the effect of lattice vibrations is likely to be important. The estimated vibrational entropies of disordering lie between $0.05 k_{B}$, for the most conservative estimates (Moraitis and Gautier, 1977), up to the order of $0.5 k_{B}$ (Tuijn and Bakker, 1989).

With the increased availability of computing power, the application of first-principles methods became a practical possibility and the unknown parameters of the theoretical models of lattice vibration have become directly computable, without relying on experimental input. Initially, only simple bulk properties, such as the bulk modulus, were computable at a reasonable cost. This prompted the development of methods to infer vibrational properties from the knowledge of elastic constants. A particularly popular scheme, the MoruzziJanak-Schwarz (MJS) method (Moruzzi et al., 1988), was used in many phase diagram calculations (Abrikosov et al., 1993; Asta et al., 1993; Becker and Sanchez, 1993; Colinet et al., 1994; Mohri, 1994; Mohri et al., 1993; Nakamura and Mohri, 1993; Oh et al., 1994; Sanchez and Becker, 1994; Sanchez et al., 1991; Tseng and Stark, 
TABLE I. Calculated vibrational entropy differences. (form): Vibrational entropy of formation from pure elements. rnd: Disordered solid solution. See Table II for other abbreviations.

\begin{tabular}{|c|c|c|c|c|c|}
\hline Composition & transition & $\begin{array}{r}\Delta S \\
\left(\frac{k_{B}}{\text { atom }}\right)\end{array}$ & $\begin{array}{c}T \\
(\mathrm{~K})\end{array}$ & Methods & Refs. \\
\hline $\mathrm{AgCu}$ & $L 1_{0}{ }^{\mathrm{a}}$ (form) & -0.11 & high & ASW,MJS & $\left(\right.$ Sanchez et al., 1991) ${ }^{b}$ \\
\hline $\mathrm{Ag}_{3} \mathrm{Cu}$ & $L 1_{2}{ }^{\mathrm{a}}$ (form) & -0.22 & high & ASW,MJS & $\left(\right.$ Sanchez et al., 1991) ${ }^{\mathrm{b}}$ \\
\hline $\mathrm{Ag}_{3} \mathrm{Cu}$ & $L 1_{2}^{\mathrm{a}}$ (form) & -0.02 & high & ASW,MJS & $\left(\right.$ Sanchez et al., 1991) ${ }^{\mathrm{b}}$ \\
\hline $\mathrm{Cu}_{3} \mathrm{Au}$ & $L 1_{2} \rightarrow$ fcc rnd & 0.12 & 663 & $\mathrm{~TB}, \mathrm{BvK}, \mathrm{QH}, \mathrm{VCA}$ & (Cleri and Rosato, 1993) \\
\hline $\mathrm{CdMg}$ & hcp rnd (form) & 0.13 & 900 & LMTO,MJS,CE & (Asta et al., 1993) \\
\hline $\mathrm{CdMg}$ & $B 19$ (form) & 0.14 & high & LMTO,MJS & $\left(\right.$ Asta et al., 1993) ${ }^{\mathrm{b}}$ \\
\hline $\mathrm{Cd}_{3} \mathrm{Mg}$ & $D 0_{19}$ (form) & -0.03 & high & LMTO,MJS & $(\text { Asta et al. }, 1993)^{\mathrm{b}}$ \\
\hline $\mathrm{CdMg}_{3}$ & $D 0_{19}$ (form) & -0.10 & high & LMTO,MJS & $($ Asta et al., 1993) b \\
\hline $\mathrm{Cu}_{3} \mathrm{Au}$ & $L 1_{2} \rightarrow$ fcc rnd & 0.10 & high & EAM,BvK,H,SC & (Ackland, 1994) \\
\hline $\mathrm{Ni}_{3} \mathrm{Al}$ & $L 1_{2} \rightarrow \mathrm{fcc} \mathrm{rnd}^{\mathrm{a}}$ & 0.29 & high & EAM,BvK,H,SC & (Ackland, 1994) \\
\hline $\mathrm{SiGe}$ & $B 3^{\mathrm{a}}$ (form) & -0.02 & high & $\mathrm{PP}, \mathrm{BvK}, \mathrm{H}$ & (Garbulsky, 1996) \\
\hline ArKr & $L 1_{0}$ (form) & -0.06 & high & pot.,BvK,H & (Garbulsky, 1996) \\
\hline $\mathrm{Ca}_{0.5} \mathrm{Mg}_{0.5} \mathrm{O}$ & fcc rnd (form) & 0.04 & high & SCPIB,BvK,H,CE & $\left(\right.$ Tepesch et al., 1996) ${ }^{\mathrm{b}}$ \\
\hline $\mathrm{Ni}_{3} \mathrm{Al}$ & $L 1_{2} \rightarrow \mathrm{fcc} \mathrm{rnd}^{\mathrm{a}}$ & 0.27 & 1400 & EAM,BvK,QH,SC & (Althoff et al., 1997) \\
\hline $\mathrm{NiCr}$ & fcc rnd (form) & n.a. & 1550 & FLASTO,VCA & (Craievich and Sanchez, 1997) \\
\hline $\mathrm{Ni}_{3} \mathrm{Al}$ & $L 1_{2} \rightarrow \mathrm{fcc} \mathrm{rnd}^{\mathrm{a}}$ & 0.20 & 1500 & EAM,MC,SC & (Morgan, 1998) \\
\hline $\mathrm{Ni}_{3} \mathrm{Al}$ & $L 1_{2} \rightarrow \mathrm{fcc} \mathrm{rnd}^{\mathrm{a}}$ & 0.22 & 1200 & EAM,MD,SC & (Ravelo et al., 1998) \\
\hline $\mathrm{Ni}_{3} \mathrm{Al}$ & $L 1_{2} \rightarrow \mathrm{fcc} \mathrm{rnd}^{\mathrm{a}}$ & 0.00 & high & $\mathrm{PP}, \mathrm{BvK}, \mathrm{QH}, \mathrm{SQS}$ & (van de Walle et al., 1998) \\
\hline $\mathrm{Ni}_{3} \mathrm{Al}$ & $L 1_{2} \rightarrow D 0_{22}^{\mathrm{a}}$ & 0.04 & high & $\mathrm{PP}, \mathrm{BvK}, \mathrm{QH}$ & (van de Walle et al., 1998) \\
\hline $\mathrm{CuAu}$ & $L 1_{0} \rightarrow \mathrm{fcc}$ rnd & 0.18 & 800 & PP,LR,QH,CE & (Ozoliņš et al., 1998c) \\
\hline $\mathrm{Cu}_{3} \mathrm{Au}$ & $L 1_{2} \rightarrow$ fcc rnd & 0.08 & 800 & PP,LR,QH,CE & (Ozolinsš et al., 1998c) \\
\hline $\mathrm{CuAu}_{3}$ & $L 1_{2} \rightarrow$ fcc rnd & 0.05 & 800 & PP,LR,QH,CE & (Ozoliņš et al., 1998c) \\
\hline $\mathrm{CuAu}$ & $L 1_{1}^{\mathrm{a}}$ (form) & 0.58 & 800 & PP,LR,QH & (Ozoliņš et al., 1998c) \\
\hline $\mathrm{CuAu}$ & $L 1_{0}$ (form) & 0.21 & 800 & PP,LR,QH & (Ozolinš̌ et al., 1998c) \\
\hline $\mathrm{Cu}_{3} \mathrm{Au}$ & $L 1_{2}$ (form) & 0.20 & 800 & PP,LR,QH & (Ozoliņš et al., 1998c) \\
\hline $\mathrm{CuAu}_{3}$ & $L 1_{2}$ (form) & 0.26 & 800 & PP,LR,QH & (Ozolinsš et al., 1998c) \\
\hline $\mathrm{Al}_{3} \mathrm{Li}$ & $L 1_{2}{ }^{\mathrm{a}} \rightarrow D 0_{22}{ }^{\mathrm{a}}$ & 0.04 & high & $\mathrm{PP}, \mathrm{BvK}, \mathrm{H}$ & (Sluiter et al., 1999) \\
\hline $\mathrm{Fe}_{3} \mathrm{Al}$ & $\mathrm{DO}_{3} \rightarrow$ bcc rnd & 0.11 & high & TB-LMTO,pot.,H,SC & (Shaojun et al., 1998) \\
\hline $\mathrm{Pd}_{3} \mathrm{~V}$ & $D 0_{22} \rightarrow$ fcc rnd & -0.07 & high & $\mathrm{PP}, \mathrm{BvK}, \mathrm{QH}, \mathrm{SQS}$ & (van de Walle and Ceder, 2000) \\
\hline $\mathrm{Pd}_{3} \mathrm{~V}$ & $L 1_{2} \rightarrow D 0_{22}$ & 0.08 & high & $\mathrm{PP}, \mathrm{BvK}, \mathrm{QH}$ & (van de Walle and Ceder, 2000) \\
\hline $\mathrm{Al}_{3} \mathrm{Sc}$ & (form) & -0.70 & high & PP,LR,QH & (Ozoliņš and Asta, 2001) \\
\hline $\mathrm{Al}_{26} \mathrm{Sc}$ & (form, per Sc atom) & 0.50 & high & PP,LR,QH & (Ozolinsš and Asta, 2001) \\
\hline $\mathrm{Al}_{2} \mathrm{Cu}$ & $\theta_{c}^{\prime} \rightarrow$ theta & 0.37 & high & PP,LR,H & (Wolverton and Ozolinš̌, 2001) \\
\hline
\end{tabular}

${ }^{\mathrm{a}}$ Metastable compound.

${ }^{\mathrm{b}}$ Calculated from the data presented in the paper.

1994). In the Cd-Mg (Asta et al., 1993), Ag-Cu (Sanchez et al., 1991), and Au-Ni (Colinet et al., 1994) systems, agreement with the experimentally measured phase diagrams was substantially improved by including vibrations in this way. In retrospect, such an improvement was to be expected because first-principles phase diagram calculations often greatly overestimate transitions temperature and any downward correction to the calculated transition temperatures yields improved agreement. The Moruzzi-Janak-Schwarz approximation nearly always yields a downward correction when ordered compounds are stiffer (softer) than the elements in ordering (phase separating) systems, a very likely situation.

More recently, other techniques were used to obtain vibrational properties from elastic constants. The so- called virtual crystal approximation (described in Appendix C) was used to calculate the vibrational free energy of a disordered alloy in the Ni-Cr system (Craievich and Sanchez, 1997). The calculated vibrational free energy exhibited qualitatively the same concentration dependence as the vibrational free energy obtained by subtracting the experimentally determined free energy from the calculated configurational free energy. The virtual crystal approximations were also used in calculations of the lattice dynamics of ordered and disordered $\mathrm{Cu}_{3} \mathrm{Au}$ (Cleri and Rosato, 1993). These calculations, which relied on a tight-binding Hamiltonian [see, for instance, Harrison (1989) and Pettifor (1992)], predicted a $0.12 k_{B}$ increase in the vibrational entropy upon disordering.

In view of the large computational requirements of accurate $a b$ initio methods, many researchers have 
TABLE II. Abbreviations used in Tables I and III.

\begin{tabular}{ll}
\hline \hline pot:: pair potentials & H: harmonic approximation \\
EAM: embedded atom method & QH: quasiharmonic approximation \\
TB: tight binding & MC: Monte Carlo \\
TB-LMTO: tight-binding LMTO & MD: molecular dynamics \\
LMTO: linear muffin-tin orbitals & D: Debye model \\
ASW: atomic spherical waves & BvK: Born-von Kármán spring model \\
PP: pseudopotential calculations & SC: supercell method \\
& LR: linear response \\
cal.: differential calorimetry measurements & VCA: virtual crystal approximation \\
1xtal: single crystal phonon dispersion measurements & MJS: Moruzzi-Janak-Schwarz model \\
INS: incoherent neutron scattering measurements & CE: cluster expansion \\
anh.: anharmonicity included. & SQS: special quasirandom structures \\
\hline \hline
\end{tabular}

sought to calculate vibrational properties with simpler energy models, whose lower computational requirements enable a more accurate handling of issues such as anharmonicity or the representation of the disordered state. The development of the embedded atom method (EAM) (Daw and Baskes, 1984) offered the opportunity to accurately model metallic alloys at a reasonable computational cost. Investigations based on the embedded atom method have typically found a large vibrational entropy change upon disordering in metallic alloys. The vibrational entropy change for $\mathrm{Cu}_{3} \mathrm{Au}$ was predicted to be $0.10 k_{B}$ (Ackland, 1994), while for $\mathrm{Ni}_{3} \mathrm{Al}$, values ranging from $0.22 k_{B}$ to $0.29 k_{B}$ were obtained (Ackland, 1994; Althoff et al., 1997, 1998; Ravelo et al., 1998). ${ }^{5}$

Other researchers constructed pair potentials from an equation of state determined from first-principles calculations (Shaojun et al., 1998). The resulting pair potentials were then used to calculate disordering vibrational entropies with no further approximations. This method attributed a vibrational entropy change of $0.11 k_{B}$ to the disordering reaction of the $\mathrm{Fe}_{3} \mathrm{Al}$ compound.

Rather unexpected results were uncovered as it became possible to compute vibrational entropy differences from a complete lattice dynamics analysis as well as state-of-the-art $a b$ initio techniques. Calculations on the Si-Ge system (Garbulsky, 1996) found almost no effect of lattice vibrations: the vibrational entropy of formation of the metastable zinc-blende structure was a mere $-0.02 k_{B}$. The first $a b$ initio calculation of a vibrational entropy of disordering (van de Walle et al., 1998) placed an upper bound of $0.05 k_{B}$ in the case of the order-disorder transition of the $\mathrm{Ni}_{3} \mathrm{Al}$ compound, in sharp contrast with previous embedded atom method calculations (Ackland, 1994; Althoff et al., 1997, 1998) which found a much larger value. Although the disagreement simply originated from a difference in the predicted volume expansion of the alloy upon disorder-

\footnotetext{
${ }^{5}$ Disordered $\mathrm{Ni}_{3} \mathrm{Al}$ is actually a metastable phase. The values quoted here are at the highest temperatures reported by the investigators, as close as possible to the true disordering temperature that would be observed if the alloy did not melt before.
}

ing, the difference between EAM and $a b$ initio results does indicate that vibrational entropy differences are very sensitive to the energy model used. In another intermetallic compound, $\mathrm{Pd}_{3} \mathrm{~V}$, the vibrational entropy change upon disordering (van de Walle and Ceder, 2000) was calculated to be $-0.07 k_{B}$, although the simplest theories put forward in the earliest investigations of lattice vibrations in alloys would predict this value to be large and positive.

In the first phase diagram calculation based on a full lattice dynamics analysis (Tepesch et al., 1996), the selfconsistent potential-induced breathing (SCPIB) method (Boyer and Mehl, 1993) was used to calculate that vibrational effects lower the top of the miscibility gap by about $10 \%$ in the $\mathrm{CaO}-\mathrm{MgO}$ system. Subsequently, firstprinciples calculations on the $\mathrm{Cu}-\mathrm{Au}$ system (Ozolinšs et al., 1998c) reported a reduction of about $20 \%$ in the transition temperatures. However, in both cases, the resulting correction on the phase boundaries decreased the agreement with the experimentally determined phase diagram, suggesting that other potential sources of error, such as the precision of the energy method used or the convergence of the cluster expansion, have to be investigated.

Very recently, two rather striking examples of systems where lattice vibrations do have an important effect and where their inclusion results in a dramatically improved agreement with experimental measurements have been uncovered. Lattice vibrations are responsible for a 27 fold increase in the solubility of scandium in aluminum (Ozolinšs and Asta, 2001), resulting in a nearly perfect agreement with experimentally determined solubility limits. Vibrational contributions were also shown (Wolverton and Ozolinšs, 2001) to be essential to correctly predict the relative stability of the different precipitates which constitute the well-known GuinierPreston zones responsible for precipitation hardening in the $\mathrm{Al}-\mathrm{Cu}$ system. It is interesting to note that these two successful examples did not require the use of a cluster expansion and did not necessitate a large number of separate first-principles calculations. Each calculation could therefore be carried out with an extremely high precision. When all other sources of errors are well controlled, the effect of lattice vibrations can be more accu- 
TABLE III. Experimental measurements of vibrational entropy differences. See Table II for abbreviations.

\begin{tabular}{|c|c|c|c|c|c|}
\hline Composition & transition & $\begin{array}{c}\Delta S \\
\left(\frac{k_{B}}{\text { atom }}\right)\end{array}$ & $\begin{array}{c}T \\
(\mathrm{~K})\end{array}$ & Methods & Refs. \\
\hline $\mathrm{Ni}_{3} \mathrm{Al}$ & $L 1_{2} \rightarrow \mathrm{fcc} \mathrm{rnd}^{\mathrm{a}}$ & 0.27 & high & cal.,D,H & (Anthony et al., 1993) \\
\hline $\mathrm{Ni}_{3} \mathrm{Al}$ & $L 1_{2} \rightarrow \mathrm{fcc} \mathrm{rnd}^{\mathrm{a}}$ & 0.19 & 343 & cal. & (Anthony et al., 1993) \\
\hline $\mathrm{Fe}_{3} \mathrm{Al}$ & $\mathrm{DO}_{3} \rightarrow$ bcc rnd & $0.10 \pm 0.03$ & high & cal.,D,H & (Nagel et al., 1997) \\
\hline $\mathrm{Cu}_{3} \mathrm{Al}$ & $L 1_{2} \rightarrow$ fcc rnd & $0.14 \pm 0.05$ & high & cal.,1xtal,H,VCA & (Nagel et al., 1995) \\
\hline $\mathrm{Fe}_{0.70} \mathrm{Cr}_{0.30}$ & bcc rnd (form) & $0.14 \pm 0.05$ & high & 1xtal,H,VCA & (Fultz, Anthony, Robertson, et al., 1995) \\
\hline $\mathrm{Fe}_{0.53} \mathrm{Cr}_{0.47}$ & bcc rnd (form) & $0.20 \pm 0.05$ & high & 1xtal,H,VCA & (Fultz, Anthony, Robertson, et al., 1995) \\
\hline $\mathrm{Fe}_{0.30} \mathrm{Cr}_{0.70}$ & bcc rnd (form) & $0.21 \pm 0.05$ & high & 1xtal,H,VCA & (Fultz, Anthony, Robertson, et al., 1995) \\
\hline $\mathrm{Ni}_{3} \mathrm{Al}$ & $L 1_{2} \rightarrow \mathrm{fcc} \mathrm{rnd}^{\mathrm{a}}$ & 0.10 & high & INS,H & (Fultz, Anthony, Nagel, et al., 1995) \\
\hline $\mathrm{Ni}_{3} \mathrm{Al}$ & $L 1_{2} \rightarrow \mathrm{fcc} \mathrm{rnd}^{\mathrm{a}}$ & 0.30 & high & INS,H,VCA & (Fultz, Anthony, Nagel, et al., 1995) \\
\hline $\mathrm{Ni}_{3} \mathrm{~V}$ & $D 0_{22} \rightarrow$ fcc rnd & $0.04 \pm 0.02$ & 300 & cal.,INS & (Nagel et al., 1996) \\
\hline $\mathrm{Co}_{3} \mathrm{~V}$ & $L 1_{2}{ }^{\mathrm{a}} \rightarrow \mathrm{fcc}$ rnd & $0.15 \pm 0.02$ & high & INS & (Nagel, Fultz, and Robertson, 1997) \\
\hline $\mathrm{Cu}_{3} \mathrm{Au}$ & $L 1_{2}$ (form) & $0.06 \pm 0.03$ & 300 & INS,anh. & (Bogdanoff et al., 1999) \\
\hline $\mathrm{Cu}_{3} \mathrm{Au}$ & $L 1_{2}$ (form) & $0.12 \pm 0.03$ & 800 & INS,anh. & (Bogdanoff et al., 1999) \\
\hline $\mathrm{Co}_{3} \mathrm{~V}$ & $h P 24 \rightarrow \mathrm{fcc}$ rnd & 0.07 & high & INS,QH & (Robertson et al., 1999) \\
\hline $\mathrm{CeSn}_{3}$ & $\gamma-\mathrm{Ce}+\beta-\mathrm{Sn} \rightarrow L 1_{2}$ & $-0.54 \pm 0.09$ & high & 1xtal,H & (Bogdanoff and Fultz, 1999) \\
\hline $\mathrm{LaSn}_{3}$ & hcp-La $+\beta-\mathrm{Sn} \rightarrow L 1_{2}$ & $-0.43 \pm 0.09$ & high & 1xtal,H & (Bogdanoff and Fultz, 1999) \\
\hline $\mathrm{Ni}_{3} \mathrm{Al}$ & $L 1_{2}$ (form) & $-0.20 \pm 0.03$ & high & 1xtal,H & (Bogdanoff and Fultz, 1999) \\
\hline $\mathrm{Ni}_{3} \mathrm{Fe}$ & $\mathrm{fcc}-\mathrm{Ni}+\mathrm{bcc}-\mathrm{Fe} \rightarrow L 1_{2}$ & $0.09 \pm 0.03$ & high & 1xtal,H & (Bogdanoff and Fultz, 1999) \\
\hline $\mathrm{Pt}_{3} \mathrm{Fe}$ & $\mathrm{fcc}-\mathrm{Pt}+\mathrm{bcc}-\mathrm{Fe} \rightarrow L 1_{2}$ & $0.14 \pm 0.03$ & high & 1xtal,H & (Bogdanoff and Fultz, 1999) \\
\hline $\mathrm{Pd}_{3} \mathrm{Fe}$ & $\mathrm{fcc}-\mathrm{Pd}+\mathrm{bcc}-\mathrm{Fe} \rightarrow L 1_{2}$ & $0.05 \pm 0.03$ & high & 1xtal,H & (Bogdanoff and Fultz, 1999) \\
\hline $\mathrm{Cu}_{3} \mathrm{Zn}$ & $\mathrm{fcc}-\mathrm{Cu}+\mathrm{hcp}-\mathrm{Zn} \rightarrow L 1_{2}$ & $-0.01 \pm 0.03$ & high & 1xtal,H & (Bogdanoff and Fultz, 1999) \\
\hline $\mathrm{Cu}_{3} \mathrm{Au}$ & $L 1_{2}($ form $)$ & $0.07 \pm 0.03$ & high & 1xtal,H & (Bogdanoff and Fultz, 1999) \\
\hline $\mathrm{Fe}_{3} \mathrm{Pt}$ & $\mathrm{bcc}-\mathrm{Fe}+\mathrm{fcc}-\mathrm{Pt} \rightarrow L 1_{2}$ & $0.55 \pm 0.03$ & high & 1xtal,H & (Bogdanoff and Fultz, 1999) \\
\hline $\mathrm{Fe}_{3} \mathrm{Al}$ & $\mathrm{bcc}-\mathrm{Fe}+\mathrm{fcc}-\mathrm{Al} \rightarrow \mathrm{DO}_{3}$ & $-0.06 \pm 0.03$ & high & 1xtal,H & (Bogdanoff and Fultz, 1999) \\
\hline
\end{tabular}

${ }^{\mathrm{a}}$ Metastable compound.

rately quantified. These examples offer good hope that $a b$ initio calculations of a complete phase diagram that include lattice vibrations can provide an accuracy comparable to experiments, provided that a well converged cluster expansion can be obtained and that highly accurate first-principles calculations are used to construct it.

\section{B. Comparison with experiments}

Over the last ten years, advances in experimental techniques have made it possible to directly measure vibrational entropy differences, instead of inferring them from discrepancies between measured thermodynamical data and calculated estimates of configurational contributions to the free energy. Experimental investigations have thus provided independent assessments of the role of lattice vibration on phase stability.

The first direct measurement was obtained from differential calorimetry measurements on the $\mathrm{Ni}_{3} \mathrm{Al}$ compound (Anthony et al., 1993) and found the vibrational entropy of disordering to be at least $0.19 k_{B}$. This finding was corroborated with subsequent incoherent neutronscattering measurements (Fultz, Anthony, Nagel, et al., 1995), which bracketed its value between $0.1 k_{B}$ and $0.3 k_{B}$. These findings fueled much of the interest of the recent theoretical literature on the $\mathrm{Ni}_{3} \mathrm{Al}$ compound
(Ackland, 1994; Althoff et al., 1997, 1998; Ravelo et al., 1998; van de Walle et al., 1998). Unfortunately, the agreement between experimental and theoretical determinations is relatively poor. Even among studies in which the magnitude of the vibrational entropy is similar, its proposed physical origin differs substantially: according to experiments, the vibrational entropy change occurred with essentially no change in volume, while most calculations (Althoff et al., 1997, 1998; Ravelo et al., 1998) attribute the vibrational entropy change almost entirely to a volume change. Many of these conflicting findings were clarified by first-principles calculations (van de Walle et al., 1998), which predicted both a very small volume change and a very small vibrational entropy change upon disordering. These results indicated that (i) the apparent large vibrational entropy change observed experimentally could very well be entirely explained by the nanocrystalline nature of the samples and the use of the virtual crystal approximation in interpreting the data and (ii) the large volume expansion upon disordering predicted by the embedded atom method is probably an artifact of the method, as it would have otherwise been clearly visible experimentally. To be fair, Fultz et al. and Althoff et al. were fully aware of these potential problems; $a b$ initio calculations merely made it possible to better quantify the errors introduced. 
The general consensus among researchers is now that given the numerous difficulties faced when studying $\mathrm{Ni}_{3} \mathrm{Al}$, unambiguous evidence of the importance of lattice vibrations should probably be sought in other systems.

Similar calorimetry measurements were then performed on the $\mathrm{Fe}_{3} \mathrm{Al}$ (Anthony et al., 1994) and $\mathrm{Cu}_{3} \mathrm{Au}$ (Nagel et al., 1995) compounds, where more conclusive results could be obtained. The vibrational entropy of disordering of $\mathrm{Fe}_{3} \mathrm{Al}$ was determined to be $0.10 k_{B}$, a result which was later corroborated by calculations (Shaojun et al., 1998). In the case of $\mathrm{Cu}_{3} \mathrm{Al}$ the experimental result, $(0.14 \pm 0.05) k_{B}$, showed very good agreement with the earlier theoretical predictions of $0.12 k_{B}$ (Cleri and Rosato, 1993). Subsequent linear-response calculations (Ozolinšs et al., 1998c) yield values ranging from 0.06 to $0.08 k_{B}$ (depending on temperature), which also compares favorably with the experimental results.

The estimation of vibrational effects was also addressed by directly probing the lattice dynamics through neutron-scattering measurements. The vibrational entropy of formation of a disordered alloy in the $\mathrm{Fe}-\mathrm{Cr}$ system was obtained from single-crystal measurements of phonon dispersion curves (Fultz, Anthony, Robertson, et al., 1995) in the virtual crystal approximation. Values ranging from $0.14 k_{B}$ to $0.21 k_{B}$ were obtained, depending on concentration.

In order to determine the lattice dynamics of disordered alloys beyond the virtual crystal approximation, the incoherent neutron-scattering technique was extensively used and refined (Bogdanoff et al., 1999; Fultz, Anthony, Nagel, et al., 1995; Nagel et al., 1996; Nagel, Fultz, and Robertson, 1997; Robertson et al., 1999). With this technique, the analysis of the experimental data is considerably simplified when the species present have comparable incoherent neutron-scattering intensities, which lead to the study of two compounds satisfying this requirement: $\mathrm{Ni}_{3} \mathrm{~V}$ and $\mathrm{Co}_{3} \mathrm{~V}$. Measurements on the $\mathrm{Ni}_{3} \mathrm{~V}$ compound (Nagel et al., 1996) found a surprisingly small vibrational entropy change upon disordering, $0.04 k_{B}$, while the $\mathrm{Co}_{3} \mathrm{~V}$ compounds exhibited a relatively large value $0.15 k_{B}$ (Nagel, Fultz, and Robertson, 1997). A related study found the vibrational entropy change associated with the fcc-hcp transition of the $\mathrm{Co}_{3} \mathrm{~V}$ compound to be $0.07 k_{B}$ (Robertson et al., 1999). It is interesting to note that the disordering reaction exhibits a larger vibrational entropy change than the allotropic transformation in $\mathrm{Co}_{3} \mathrm{~V}$. Perhaps more importantly, the investigations of the $\mathrm{Ni}_{3} \mathrm{~V}$ and $\mathrm{Co}_{3} \mathrm{~V}$ compounds presented the first experimental evidence of important anharmonic effects.

The same technique of incoherent neutron scattering was employed to revisit the $\mathrm{Cu}-\mathrm{Au}$ system (Bogdanoff et al., 1999). The vibrational entropy of formation of the $\mathrm{Cu}_{3} \mathrm{Au}$ compound was found to be $0.06 k_{B}$ at $300 \mathrm{~K}$, corroborating earlier estimations based on phonon dispersion curve measurements (Bogdanoff and Fultz, 1999). At $800 \mathrm{~K}$, the measured value of $(0.12 \pm 0.04) k_{B}$ is consistent with the value of $0.20 k_{B}$ obtained with $a b$ initio calculations (Ozolinšs et al., 1998c).
The vibrational entropy of formation of various ordered compounds, obtained from single-crystal phonon dispersion measurements, were recently compiled (Bogdanoff and Fultz, 1999) and show formation values of up to $0.5 k_{B}$. However, this compilation contains many systems where the alloy has a crystal structure that differs from the one of the pure elements and the formation values thus also include the vibrational entropy change associated with a structural transition. When all these cases are excluded, the maximum vibrational entropy change decreases to a more conservative upper bound of $0.20 k_{B}$, which is reached in the ordered phase of $\mathrm{Ni}_{3} \mathrm{Al}$.

\section{Conclusion}

Although early investigations of the impact of vibrational effects on phase stability consistently found large effects, it is now becoming apparent, as more precise theoretical and experimental techniques became available, that vibrational effects are often, but not always, large. It is therefore important to identify the factors which determine when they are, so that the effort devoted to calculating them is proportional to their expected magnitude.

\section{THE ORIGIN OF VIBRATIONAL ENTROPY DIFFERENCES BETWEEN PHASES}

We have presented the framework that allows for the inclusion of vibrational effects in phase diagram calculations. However, the formalism presented so far does not directly provide any intuition regarding the origin of vibrational entropy differences. This intuition is important to be able the predict when vibrational effects should be important and, when they are, which approximation should be used to calculate them.

Three mechanisms have been suggested to explain the origin of vibrational entropy differences in alloys. We will discuss them in turn.

\section{A. The "bond proportion" effect}

In most theoretical studies based on simple models systems (Bakker, 1982a; Bakker and Tuijn, 1986; Garbulsky and Ceder, 1994; Matthew et al., 1983; Tuijn and Bakker, 1989), the effect of the state of order of an alloy on its vibrational entropy has been attributed to the fact that bonds between different chemical species have a different stiffness than the bonds between identical species. When the proportion of each type of bond in the alloy changes during, for instance, an order-disorder transition, the average stiffness of the alloy changes as well, resulting in a change of its vibrational entropy. This so-called bond proportion mechanism is illustrated in Fig. 2 in the case of an order-disorder transition. In a system with ordering tendencies, the bond between unlike atoms are associated with an increased stability and are thus expected to be stiffer than bonds between like atoms. Since disordering reduces the number of bonds 


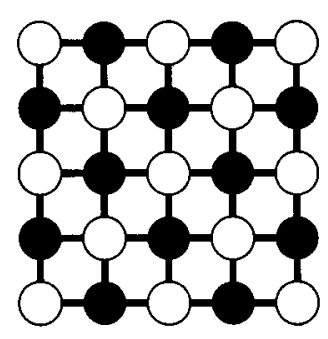

Ordered

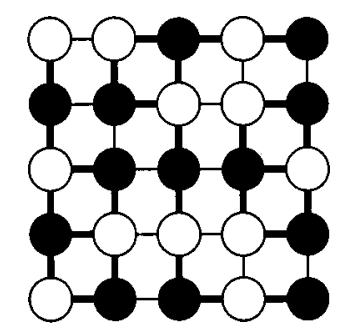

Disordered

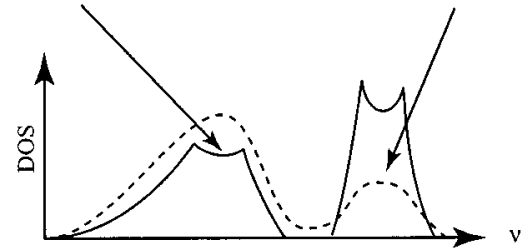

FIG. 2. The bond proportion mechanism. Ordered alloys are characterized by the fact that a large proportion of the nearestneighbor bonds join unlike atoms. As these types of bonds are presumably stiffer, they are responsible for an increased density of high-frequency optical-phonon modes. Disordering reduces the proportion of stiff nearest-neighbor bonds, and the density of high-frequency optical modes decreases correspondingly, resulting in an increase in vibrational entropy.

between unlike atoms in favor of bonds between similar atoms, the disordered state is expected to be softer, and thus have a large vibrational entropy. Vibrations would then tend to stabilize the disordered state relative to the ordered state, reducing the transition temperature. A similar reasoning in the case of a phase separating system shows that the disordered state should be softer than a phase separated mixture, indicating that the miscibility gap should be lowered as a result of vibrational effects.

The presence of a bond proportion effect can be readily identified from the nature of the changes taking place in the phonon densities of states during an orderdisorder transition. In the ordered alloy, the very stiff nearest-neighbor bonds should be associated with highfrequency optical modes peaks. As the alloy disorders, the height of these peaks should decrease since the number of stiff bonds decreases. This characteristic signature of the bond proportion mechanism in the phonon DOS has been repeatedly observed in experiments (Anthony et al., 1994; Fultz, Anthony, Nagel, et al., 1995) as well as in theoretical calculations (Althoff et al., 1997) (although, in the latter study the bond proportion mechanism was dominated by other effects discussed below).

\section{B. The volume effect}

It is well known that vibrational entropy of a given compound varies with volume: This dependence is responsible for thermal expansion. It is thus expected that the volume change that typically occurs during solidstate transitions should also result in a change in vibrational entropy. As the alloy expands (or contracts), as a result of a change in its state of order, the stiffness of all
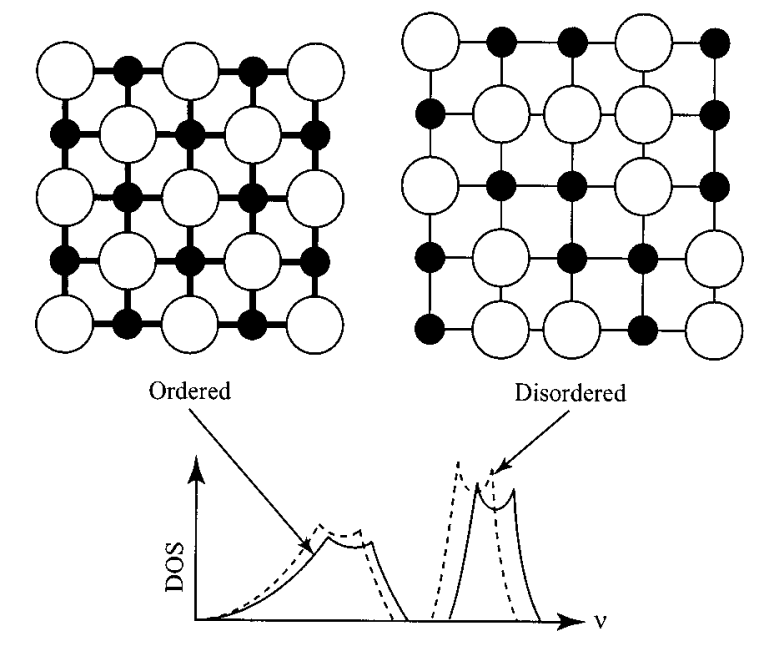

Disordered

FIG. 3. The volume mechanism. Disordering is typically associated with an increase in volume. Since chemical bonds tend to soften as they lengthen, an overall increase in volume should be associated with a corresponding decrease in the frequency of all phonon modes, resulting in an increase in vibrational entropy.

chemical bonds decreases (or increases). The resulting change in vibrational entropy is entirely due to anharmonicity, in contrast to the bond proportion effect. When the volume mechanism operates alone, the phonon DOS should exhibit an overall shift when the volume changes (see Fig. 3).

This shift is usually accompanied by a change in the shape of the phonon DOS, so that visual inspection of the phonon DOS is usually not sufficient to identify the shift due to the effect of volume. Theoretical investigations of this effect have thus relied on a simple thought experiment consisting in separating the vibrational entropy change upon disordering at constant volume from the vibrational entropy change resulting solely from the volume expansion of the disordered state. In this fashion, the importance of volume changes was first observed in embedded atom method (EAM) calculations of disordering reaction of the $\mathrm{Ni}_{3} \mathrm{Al}$ and $\mathrm{Cu}_{3} \mathrm{Au}$ compounds (Ackland, 1994) and later corroborated by subsequent embedded atom method calculations on the $\mathrm{Ni}_{3} \mathrm{Al}$ compound (Althoff et al., 1997; Ravelo et al., 1998). These more recent calculations also found that the volume effect is magnified by the fact that the linear thermal expansion coefficient of different phases can differ substantially (by about $5 \times 10^{-6} \mathrm{~K}^{-1}$ relative to an absolute value of about $\left.15 \times 10^{-6} \mathrm{~K}^{-1}\right)$. First-principles calculations on the $\mathrm{Cu}-\mathrm{Au}$ system (Ozolinšs et al., 1998c) revealed a similar finding. In contrast, first-principles calculations on the $\mathrm{Ni}_{3} \mathrm{Al}$ (van de Walle et al., 1998) and $\mathrm{Pd}_{3} \mathrm{~V}$ (van de Walle and Ceder, 2000) compounds found only a small difference between the thermal-expansion coefficients of the ordered and the disordered phases (less than $1 \times 10^{-6} \mathrm{~K}^{-1}$ ).

Even though the temperature dependence of vibrational entropy can be large, it is important to keep in mind that a given vibrational entropy difference arising from anharmonic contributions has a smaller impact on 
the vibrational free energy than a harmonic contribution of the same magnitude. The reason is that anharmonic contributions to the vibrational entropy are always partly canceled by anharmonic contributions to the vibrational enthalpy. In contrast, such cancellation does not occur for harmonic contributions. ${ }^{6}$ The quasiharmonic approximation predicts that anharmonic contributions have exactly half the effect of harmonic contributions (see Ozolinšs et al., 1998c or Appendix B) when the volume dependence of the energy is quadratic while the volume dependence of the vibrational entropy is linear.

Experimental measurements have not identified the volume mechanism as a major source of entropy differences since the simple thought experiment that allows its identification in calculations cannot be performed experimentally. The effect of thermal expansion on the phonon DOS, however, is clearly seen experimentally (Nagel et al., 1996; Nagel, Fultz, and Roberston, 1997), due to the fact that thermal expansion causes shifts in the phonon DOS that are not accompanied by substantial changes in its shape. Measurements on $\mathrm{Ni}_{3} \mathrm{~V}$ (Nagel et al., 1996), $\mathrm{Co}_{3} \mathrm{~V}$ (Nagel, Fultz, and Robertson, 1997), and $\mathrm{Cu}_{3} \mathrm{Au}$ (Bogdanoff et al., 1999) all show that anharmonic contributions are not negligible.

\section{The size mismatch effect}

The third advocated source of vibrational entropy changes is the effect of atomic size mismatch. When atoms of different sizes are constrained to coexist on a lattice, the atoms can experience compressive (or tensile) stress that results in locally stiffer (or softer) regions. When large atoms sit on neighboring lattice sites, the amplitude of their vibrations is reduced, i.e., the alloy tends to be locally stiffer. Conversely, when small atoms sit on neighboring lattice sites, the extra room available results in a locally softer region.

The phenomenon was first noted in EAM calculations on the $\mathrm{Cu}_{3} \mathrm{Au}$ compound (Auckland, 1994), where, in the disordered state, the presence of highly compressed pairs of $\mathrm{Au}$ atoms lowers the vibrational entropy of the disordered state. A similar effect was found in firstprinciples calculations on $\mathrm{Ni}_{3} \mathrm{Al}$ (van de Walle et al., 1998), where very compressed pairs of $\mathrm{Al}$ atoms were

\footnotetext{
${ }^{6} \mathrm{~A}$ temperature dependence of vibrational entropy necessarily introduces a temperature dependence of the vibrational enthalpy, as a consequence of the following thermodynamic relation: $\partial H_{\mathrm{vib}} / \partial T=T\left(\partial S_{\mathrm{vib}} / \partial T\right)$. The anharmonic vibrational free energy is then a sum of two competing contributions: $F_{\text {vib }}=H_{\text {vib }}-T S_{\text {vib }}$. In contrast, harmonic contributions to the vibrational enthalpy are configuration independent in the hightemperature limit, by the equipartition theorem, and give no net contribution to vibrational free-energy differences. Vibrational entropy differences originating from harmonic contributions thus enter the expression for vibrational free-energy differences directly, without any partial cancellation from the enthalpic term.
}

found in the disordered state. In first-principles calculations on $\mathrm{Pd}_{3} \mathrm{~V}$ (van de Walle and Ceder, 2000), an even more intriguing size-related effect was observed: all three types of chemical bonds have incompatible equilibrium lengths and the vibrational entropy changes can be explained solely by the large relaxations of the atoms away from their ideal lattice sites in the disordered state. A summary of these observations can be found in Morgan et al. (2000).

In the experimental literature, the size mismatch effect has been described with the help of a stiff sphere picture first introduced in an investigation of the $\mathrm{Cu}_{3} \mathrm{Au}$ compound (Nagel et al., 1995). The fundamental intuition behind this picture is that a chemical bond becomes stiffer when the two bonded atoms have touching atomic "spheres." Further evidence for this stiff sphere picture was provided by a systematic analysis of the vibrational entropy of formation of various $L 1_{2}$ compounds (Bogdanoff and Fultz, 1999), which shows a correlation with the difference in radii between the two alloyed species.

\section{COMPUTATIONAL TECHNIQUES}

To understand the nature of the difficulties encountered, it is instructive to first consider how, in principle, the vibrational properties of a single configuration $\sigma$ can be calculated with an arbitrary accuracy. The techniques presented in this section are the tools that were used to investigate the importance of lattice vibrations presented in Sec. III.A.

Phase diagram calculation involves computing vibrational properties for a set of configurations $\sigma$. Carrying out the full phonon problem for each configuration results in undue computational requirements. Nevertheless, the formal solutions presented here play an important role in devising practical ways to include vibrational effects in phase diagram calculations. This section first focuses on the treatment lattice vibration within the harmonic approximation, before addressing the issue of anharmonicity. Finally, important consideration concerning the energy models used as an input for these procedures are discussed.

\section{A. Lattice vibrations in the harmonic approximation}

In this section, we review the problem of determining the constrained free energy of a system in the neighborhood of a configuration $\sigma$, under the assumption that the system spends most of its time in a region near a local energy minimum, where a harmonic approximation to the energy surface is accurate. In this approximation, the free energy determination reduces to the well-known phonon problem (Ashcroft and Mermin, 1976; Maradudin et al., 1971).

\section{Theory}

Consider a system consisting of $N$ atoms. Let $M_{i}$ be the mass of atom $i$ and $u(i)$ be its displacement away from its equilibrium position. Time derivatives are de- 
noted by dots while greek letter subscripts denote one of the Cartesian components of a vector. In the harmonic approximation, the energy of the system can be written as

$$
H=\frac{1}{2} \sum_{i} M_{i}[\dot{u}(i)]^{2}+\frac{1}{2} \sum_{i, j} u^{T}(i) \Phi(i, j) u(j),
$$

where

$$
\Phi_{\alpha \beta}(i, j)=\left.\frac{\partial^{2} E}{\partial u_{\alpha}(i) \partial u_{\beta}(j)}\right|_{u(l)=0 \forall l} .
$$

The $3 \times 3$ matrices $\Phi(i, j)$ are called the force constants tensors, as they relate the displacement of atom $j$ to the force $f$ exerted on atom $i$ :

$$
f(i)=\Phi(i, j) u(j) .
$$

Such a harmonic approximation of a solid is often referred to as a Born-von Kármán model.

Note that contrary to usual treatment, we do not immediately impose translational symmetry, in order to derive a few general results that also apply to systems such as disordered alloys.

The substitution $e(i)=\sqrt{M_{i}} u(i)$ yields

$$
H=\frac{1}{2}\left(\sum_{i} \dot{e}^{2}(i)+\sum_{i, j} e^{T}(i) \frac{\Phi(i, j)}{\sqrt{M_{i} M_{j}}} e(j)\right) .
$$

The $3 N$ eigenvalues $\lambda_{m}$ of the matrix, ${ }^{7}$

$$
D=\left(\begin{array}{ccc}
\frac{\Phi(1,1)}{\sqrt{M_{1} M_{1}}} & \cdots & \frac{\Phi(1, N)}{\sqrt{M_{1} M_{N}}} \\
\vdots & \ddots & \vdots \\
\frac{\Phi(N, 1)}{\sqrt{M_{N} M_{1}}} & \cdots & \frac{\Phi(N, N)}{\sqrt{M_{N} M_{N}}}
\end{array}\right),
$$

then give the frequencies $\nu_{m}=(1 / 2 \pi) \sqrt{\lambda_{m}}$ of the normal modes of oscillation. In the harmonic approximation, the knowledge of these frequencies is sufficient to determine the thermodynamic quantities we are interested in. This information is conveniently summarized by the phonon density of states (DOS), which gives the number of modes of oscillation having a frequency lying in the interval $[\nu, \nu+d \nu]$ :

$$
g(\nu)=\frac{1}{N} \sum_{m=1}^{3 N} \delta\left(\nu-\nu_{m}\right) .
$$

It can be shown that the free energy of the system (restricted to remain close to a given configuration $\sigma$ ) is given by (Maradudin et al., 1971)

\footnotetext{
${ }^{7}$ Among the $3 N$ eigenvalues, the six eigenvalues associated with rigid body translations and rotations are zero. In the thermodynamic limit, these few degrees of freedom are inconsequential. To avoid notational complications, we simply assume that the solid is fixed to a reference frame by springs so that the resulting dynamical matrix has $3 N$ nonzero eigenvalues.
}

$$
\begin{aligned}
\frac{F}{N} & =\frac{E^{*}}{N}+k_{B} T \int_{0}^{\infty} \ln \left[2 \sinh \left(\frac{h \nu}{2 k_{B} T}\right)\right] g(\nu) d \nu \\
& =\frac{E^{*}}{N}+\frac{k_{B} T}{N} \sum_{m} \ln \left[2 \sinh \left(\frac{h \nu_{m}}{2 k_{B} T}\right)\right],
\end{aligned}
$$

where $E^{*}$ is the potential energy of the system at its equilibrium position and $h$ is Planck's constant. Phase transitions in alloys typically occur at a temperature where the high-temperature limit of this expression is an accurate approximation:

$$
\begin{aligned}
\frac{F}{N} & =\frac{E^{*}}{N}+k_{B} T \int_{0}^{\infty} \ln \left(\frac{h \nu}{k_{B} T}\right) g(\nu) d \nu \\
& =\frac{E^{*}}{N}+\frac{k_{B} T}{N} \sum_{m} \ln \left(\frac{h \nu_{m}}{k_{B} T}\right) .
\end{aligned}
$$

The usual criterion used to determine the temperature range where high-temperature limit is reached is the Debye temperature. Note that the factor $h / k_{B} T$ is often omitted because it cancels out when calculating vibrational free-energy differences. In the high-temperature limit, another important form of cancellation occurs: The atomic masses have no effect on the free energies of formation (Garbulsky and Ceder, 1994; Grimvall and Rosen, 1983). This important result, shown in Appendix A, rules out that masses play any significant role in determining phase stability at high temperatures.

As mentioned before, a convenient measure of the magnitude of the effect of lattice vibrations on phase stability is the vibrational entropy, which can be obtained from the vibrational free energy by the wellknown thermodynamical relationship $S_{\mathrm{vib}}=-\partial F_{\mathrm{vib}} / \partial T$. Contrary to the vibrational free energy of formation, the vibrational entropy of formation ${ }^{8}$ is temperature independent in the high-temperature limit of the harmonic approximation, allowing a unique number to be reported as a measure of the importance of vibrational effects.

In a crystal, the determination of the normal modes is somewhat simplified by the translational symmetry of the system. Let $n$ denote the number of atoms per unit cell. Let $u\left(\begin{array}{l}l \\ i\end{array}\right)$ denote the displacements of atom $i$ in cell $l$ away from its equilibrium position. Let $\Phi\left(\begin{array}{l}l l_{i j}^{\prime} \\ l^{\prime}\end{array}\right)$ be the force constant relative to atom $i$ in cell $l$ and atom $j$ in cell $l^{\prime}$ and let $e\left(\begin{array}{l}l \\ i\end{array}\right)=\sqrt{M_{i}} u\left(\begin{array}{l}l \\ i\end{array}\right)$. Bloch's theorem indicates that the eigenvectors of the dynamical matrix are of the form

$$
e\left(\begin{array}{l}
l \\
i
\end{array}\right)=\exp [\iota 2 \pi(k \cdot l)] e\left(\begin{array}{l}
0 \\
i
\end{array}\right),
$$

where $\iota=\sqrt{-1}, l$ denotes the Cartesian coordinates of one corner of cell $l$, and $k$ is a point in the first Brillouin

\footnotetext{
${ }^{8}$ The absolute value of the vibrational entropy is not constant at high temperature, but its temperature dependence does not vary across distinct phases and thus formation values are temperature independent.
} 
zone. This fact reduces the problem of diagonalizing the $3 N \times 3 N$ matrix $D$ to the problem of diagonalizing a $3 n \times 3 n$ matrix $D(k)$ for various values of $k$. This can be shown by a simple substitution of Eq. (16) into Eq. (13). ${ }^{9}$ The dynamical matrix $D(k)$ to be diagonalized is given by ${ }^{10}$

$$
D(k)=\sum_{l} \exp [\iota \pi(k \cdot l)]\left(\begin{array}{ccc}
\frac{\Phi\left(\begin{array}{ll}
0 & l \\
1 & 1
\end{array}\right)}{\sqrt{M_{1} M_{1}}} & \cdots & \frac{\Phi\left(\begin{array}{ll}
0 & l \\
1 & n
\end{array}\right)}{\sqrt{M_{1} M_{n}}} \\
\vdots & \ddots & \vdots \\
\frac{\Phi\left(\begin{array}{ll}
0 & l \\
n & 1
\end{array}\right)}{\sqrt{M_{n} M_{1}}} & \cdots & \frac{\Phi\left(\begin{array}{ll}
0 & l \\
n & n
\end{array}\right)}{\sqrt{M_{n} M_{n}}}
\end{array}\right) .
$$

As before, the resulting eigenvalues $\lambda_{i}(k)$ for $i$ $=1, \ldots, n$ give the frequencies of the normal modes $\left[\nu_{i}(k)=(1 / 2 \pi) \sqrt{\lambda_{i}(k)}\right]$. The function $\nu_{i}(k)$ for a given $i$ is called a phonon branch, while the plot of the $k$ dependence of all branches along a given direction in $k$ space is called the phonon dispersion curve. In periodic systems, the phonon DOS is defined as

$$
g(\nu)=\sum_{i=1}^{3 n} \int_{B Z} \delta\left[\nu-\nu_{i}(k)\right] d k,
$$

where the integral is taken over the first Brillouin zone.

\section{Force-constant determination}

The above theory relies, of course, on the availability of the force-constant tensors. The determination of these force constant tensors is the focus of this section. Before describing the methods used for their determination, we will first review important properties of the force constant tensors.

While the number of unknown force constants to be determined is in principle infinite, it can, in practice, be reduced to a manageable finite number with the help of the following two observations. First, the force constant $\Phi(i, j)$ between two atoms $i$ and $j$ beyond a given distance can be neglected. Second, the symmetry of the crystal imposes linear constraints between the elements of the force-constant tensors.

The accuracy of the approximation made by truncating the range of force constant can be tested by gradually increasing the range of interactions, until the quantities to be determined no longer vary substantially. It is important to note that most thermodynamic quantities can be written as a weighted integral of the phonon DOS and their convergence rates are thus much faster

\footnotetext{
${ }^{9}$ The summation over atoms needs to be replaced by summations over atoms and cells.

${ }^{10}$ The reader should be aware that there are many possible conventions regarding the phase factor: for instance, $\exp [\iota 2 \pi(k \cdot l)], \quad \exp [-\imath 2 \pi(k \cdot l)], \quad \exp [\iota(k \cdot l)], \quad \exp (\iota 2 \pi\{k \cdot[l$ $+x(j)]\})$ where $x(j)$ is the coordinate of atom $j$ within the cell. While all conventions yield different dynamical matrices, they all have the same eigenvalues.
}

than the pointwise convergence rate of the phonon DOS itself (Garbulsky, 1996; van de Walle et al., 1998). That is, the errors on the DOS at each frequency tend to be quickly averaged out when the contributions of each frequency are added.

The restrictions on the force constants imposed by the symmetry of the lattice can be expressed as follows. Consider the force constant $\Phi(i, j)$ of atoms $i$ and $j$ located at $x(i)$ and $x(j)$ and consider a symmetry transformation that maps a point of coordinate $x$ to $S x+t$, where $S$ is a $3 \times 3$ matrix and $t$ and $3 \times 1$ translation vector. In general, if the crystal is left unchanged by such a symmetry operation, the force-constant tensors should be left unchanged as well. This fact imposes the following constraints on the spring tensors:

$$
\left.\begin{array}{l}
S x(i)+t=x\left(i^{\prime}\right) \\
S x(j)+t=x\left(j^{\prime}\right)
\end{array}\right\} \Rightarrow \Phi(i, j)=S^{T} \Phi\left(i^{\prime}, j^{\prime}\right) S .
$$

Additional constraints on the force constants can be derived from simple invariance arguments. The most important constraints, obtained by noting that rigid translations and rotations must leave the forces exerted on the atoms unchanged, are

$$
\begin{aligned}
& \Phi(i, i)=-\sum_{j \neq i} \Phi(i, j), \\
& \Phi(i, j)=\Phi^{T}(j, i) .
\end{aligned}
$$

Additional constraints can be found in Maradudin et al. (1971) and Born and Huang (1956).

There are essentially three approaches to determining the force constants: analytic calculations, supercell calculations, and linear-response calculations. Analytic calculations are only possible when the energy model is sufficiently simple to allow a direct calculation of the second derivatives of the energy with respect to atomic displacements, as in the case of empirical pair potential models. For first-principles calculations, either one of the two following methods have to be used.

a. The supercell method. The supercell method (Wei and Chou, 1992; Garbulsky, 1996) consists of slightly perturbing the positions of the atoms away from their equilibrium position and calculating the reaction forces. Equating the calculated forces to the forces predicted from the harmonic model yields a set of linear constraints that allows the unknown force constants to be determined, ${ }^{11}$

$$
F(i)=\Phi(i, j) u(j) .
$$

\footnotetext{
${ }^{11}$ Equalities between calculated and predicted energies can be used as well. Using energies alone to determine the force constants would be a rather inefficient use of the information provided by $a b$ initio calculations. Once a first-principles calculation of the energy of a distorted structure has been completed, the calculation of the forces acting on the atoms is computationally inexpensive. The knowledge of the energy provides a single equation while the knowledge of the forces provides up to three equations per atom.
} 
When the force constants considered have a range that exceeds the extent of the primitive cell, a supercell of the primitive cell has to be used. (The simultaneous movement of the image atoms introduces linear constrains among the forces that prevent the determination of some of the force constants.)

While any choice of the perturbations that allows the force constants to be determined is in principle equally valid, a few simple principles drastically narrow down the number of perturbations that need to be considered. For a given supercell, there is only a finite number of nonredundant perturbations to consider.

A minimal set of nonredundant perturbations can be obtained as follows.

- Consider in turn each atom in the asymmetric unit of the primitive cell.

- Mark the chosen atom (and its periodic images in the other supercells) and consider it as distinct from other atoms of the same type. (This operation effectively removes some of the symmetry operation of the space group of the crystal.)

- Construct the point group $\left\{S_{i}\right\}$ of the site where this atom is located. ( $S_{i}$ is a $3 \times 3$ matrix.)

- Move the chosen atom along a direction $u_{1}$ such that the space spanned by the vectors $S_{i} u_{1}$ (for all $i$ ) has the highest dimensionality possible.

- If the resulting dimensionality is less than three, consider an additional direction $u_{2}$ such that the space spanned by the vectors $S_{i} u_{j}$ for $j=1,2$ has the highest dimensionality possible.

- If the resulting dimensionality is less than three, consider a direction $u_{3}$ orthogonal to $u_{1}$ and $u_{2}$.

The resulting displacements $u_{j}$ for all atoms in the asymmetric unit give a minimal list of perturbations that is sufficient to find all the force constants that can possibly be determined with the given supercell. This result follows from the observation that any other possible displacement can be written as a linear combination of the displacements considered above (or displacements that are symmetrically equivalent to them).

When determining force constants with the supercell method, it is important to verify that the presence of small numerical noise in the calculated forces does not result in too much error in the fitted force constants. To minimize noise in the fitted force constants, it may be necessary to use more than the minimum possible number of perturbations. The additional perturbations should ideally be based on different supercells, to minimize the systematic errors introduced by the movement of the image atoms.

When $a b$ initio calculations are used to calculate the forces, it is especially important to iterate the electronic self-consistency steps to convergence. Even though the energy may appear to be well converged, the forces may not yet be. Energy is the solution to a minimization procedure, while forces are not. As a result, errors on the energy are of a second order in the minimization parameters, while the errors on the forces are of the first order in the minimization parameters. For the same reason, special attention should be given to the structural relaxations.

The true system is not exactly harmonic and the calculated forces may exhibit anharmonic components that introduce noise into the fitted force constants. This problem can be alleviated by considering an additional set of perturbations, where the displacements have the opposite sign. Subtracting the calculated forces obtained for this new set of displacements from the corresponding displacements of the opposite sign exactly cancels out all the odd-order anharmonic terms. Of course, for perturbations such that the negative displacement is equivalent by symmetry to the corresponding positive displacement, this duplication is unnecessary, because the terms of odd order are already zero by symmetry.

Additional guidelines for fitting force constants can be found in Ackland et al. (1997), Wei and Chou (1992), and Garbulsky (1996).

b. Linear response. Linear-response calculations seek to directly evaluate the dynamical matrix for a set of $k$ points. The starting point of the linear-response approach is evaluation of the second-order change in the electronic energy induced by atomic displacements from perturbation theory. Within this framework, practical schemes to compute vibrational properties in semiconductors (Baroni et al., 1987; Giannozzi et al., 1991; Gonze et al., 1992; Waghmare, 1996) and metallic systems (de Gironcoli, 1995; Ozolinšs, 1996; Quong and Klein, 1992) have been devised. In this section we will not discuss the theory behind linear response calculations which can be found in a recent review (Gonze, 1997; Gonze and Lee, 1997), but rather focus on how the results of linear-response calculations can be used in the context of alloy phase diagram calculations.

The dynamical matrices calculated from linearresponse theory are exact in the sense that they account for arbitrarily long-range force constants. While in the supercell method inaccuracies arise from the truncation of the force constants, the limit in precision for linear response calculations arises from the use of a small number of $k$ points to sample the Brillouin zone. To address this issue, two methods can be used.

A set of special $k$ points can be selected through the Chadi-Cohen (Chadi and Cohen, 1973) or MonkhorstPack (Monkhorst and Pack, 1976) schemes. Special $k$ points are selected so that the integral over the Brillouin zone of a function $h(k)$ that contains no Fourier components above a given frequency can be exactly evaluated by a weighted average of the function at each special point. Since thermodynamic quantities can be written as integrals of functions of the dynamical matrix $f(D(k))$ over the Brillouin zone, the procedure is straightforward to apply in this context.

The other approach is the so-called Fourier inversion method [see, for instance, Giannozzi et al. (1991) and Quong and Klein (1992)]. The calculated dynamical matrices from a set of $k$ points are used to determine the value of the force constants up to a certain interaction range. The resulting harmonic model can then be used 
to calculate the dynamical matrix at any point in the Brillouin zone, allowing a much finer sampling of the Brillouin zone for the purpose of performing the numerical integration required to determine any thermodynamic quantity.

The Fourier inversion method is preferable when the function $f(D(k))$ to be integrated exhibits highfrequency components, while the dynamical matrix itself, $D(k)$, does not. Such a situation would arise when the function $f$ is highly nonlinear. The smoothness of $D(k)$ then ensures that it can be represented with a small number of Fourier components. The less wellbehaved function $f(D(k))$ can then be accurately integrated with as many $k$ points as needed, using the dynamical matrix $D(k)$ calculated from the spring model.

In the case of vibrational free-energy calculations, the special $k$ points method has been observed to converge rapidly with respect to the number of $k$ points (Garbulsky and Ceder, 1996), ${ }^{12}$ so that the Fourier inversion method is probably unnecessary. ${ }^{13}$

For a given set of special $k$ points, there is an approximate correspondence between the number of Fourier components that can be integrated exactly and the range of force constants that can be determined. The correspondence is exact only when the lattice has one atom per cell and when the function $f$ is linear. ${ }^{14}$

While supercell and linear-response calculations are in principle equivalent in terms of the information they provide, they have complementary advantages in terms of computational efficiency. The linear-response method is the most efficient way to perform high-accuracy calculations that would otherwise be tedious and computer intensive with the supercell method. However, when a high accuracy is not needed, the supercell method has the advantage that various simplifying assumptions regarding the structure of the force constant tensors can transparently be used to drastically reduce computational requirements. It is not clear at which level of accuracy the crossover between the efficiency of each approach occurs, but it is important to keep both approaches in mind. Another consideration is that in the continuously evolving field of computational solid-state physics, new first-principles energy methods are continually developed, and the derivation of the appropriate linear-response theory always follows the derivation of simple force calculations. Hence, despite the elegance of

\footnotetext{
${ }^{12}$ Calculations of the authors, based on data from Ozolinšs et al. (1998c) also support this finding.

${ }^{13}$ Note that the function whose integral gives the free energy exhibits a logarithmic singularity at the $\Gamma$ point, which could lead to high-frequency components that are difficult to integrate accurately. However, in three-dimensional systems, this logarithmic singularity contributes very little to the value of the free energy, so that the rate of convergence of the integral as a function of the number of $k$ points is not dramatically slowed down by the presence of the singularity. As a result, the special $k$ point method can safely be used in practical calculations of the vibrational free energy.

${ }^{14}$ As can be shown by a simple Fourier transform.
}

linear-response theory, it is to be expected that the supercell method will always remain of interest.

\section{B. Anharmonicity}

While the harmonic approximation is remarkably accurate given its simplicity, it has one important limitation: It is unable to model thermal expansion and its impact on vibrational properties. Both the free energy $F$ and the entropy $S$ can be obtained from the the heat capacity $C_{p}$ :

$$
S=\int_{0}^{T} \frac{C_{p}}{T} d T \text { and } F=\left.E\right|_{T=0}-\int_{0}^{T} S d T,
$$

Hence a simple way to account for thermal expansion is to use the following well-known thermodynamic relationship between the heat capacity at constant pressure $C_{p}$ and at constant volume $C_{v}$ :

$$
C_{p}=C_{v}+B V T \alpha^{2},
$$

where $\alpha$ is the coefficient of volumetric thermal expansion while $B$ is the bulk modulus. In a purely harmonic model, there is no thermal expansion and $C_{v}$ is equal to $C_{p}$. The term $B V T \alpha^{2}$ can thus be viewed as correction arising from anharmonic effects.

Equation (22) is directly useful in the context of experimental measurements where $C_{p}, V$, and $\alpha$ can be directly measured (Nagel et al., 1996; Robertson et al., 1999). In the following section, we describe the computational techniques used to handle anharmonicity.

\section{The quasiharmonic model}

A simple modification to the harmonic approximation, called the quasiharmonic approximation, allows the calculation of thermal expansion at the expense of a moderate increase in computational cost. In the quasiharmonic approximation, the phonon frequencies are allowed to be volume dependent, which amounts to assuming that the force constant tensors are volume dependent [see, for instance, Grimvall (1986)]. This approximation has recently been shown to be extremely reliable, enabling accurate first-principles calculations of the thermal-expansion coefficients of many elements up to their melting points (Quong and Lui, 1997).

The best way to understand this approximation is to study a simple model system where it is essentially exact. Consider a linear chain (with periodic boundary conditions) of identical atoms interacting solely with their nearest neighbors through a pair potential of the form

$$
U(r)=a_{1} r+a_{2} r^{2}+a_{3} r^{3} .
$$

Let $L$ be the average distance between two nearest neighbors and let $u(i)$ denote the displacement of atom $i$ away from its equilibrium position. The total potential energy (per atom) of this system is then given by

$$
\begin{aligned}
\frac{U}{N}= & \frac{1}{N} \sum_{i} a_{1}[L+u(i)-u(i+1)]+a_{2}[L+u(i) \\
& -u(i+1)]^{2}+a_{3}[L+u(i)-u(i+1)]^{3} .
\end{aligned}
$$


This expression can be simplified by noting that all the terms that are linear in $[u(i)-u(i+1)]$ cancel out when summed over $i$,

$$
\begin{aligned}
\frac{U}{N}= & a_{1} L+a_{2} L^{2}+a_{3} L^{3}+\left(a_{2}+3 a_{3} L\right) \\
& \times \frac{1}{N} \sum_{i}[u(i)-u(i+1)]^{2}+O\left\{[u(i)-u(i+1)]^{3}\right\} .
\end{aligned}
$$

The first three terms, $a_{1} L+a_{2} L^{2}+a_{3} L^{3}$, give the elastic energy of a motionless lattice while the remaining terms account for lattice vibrations. The important feature of this equation is that, even within the harmonic approximation, the prefactor of the harmonic term $\left(a_{2}\right.$ $\left.+3 a_{3} L\right)$ depends on the anharmonicity of the potential (through $a_{3} L$ ). In the more realistic case of threedimensional systems, this length dependence translates into a volume dependence ${ }^{15}$ of the harmonic force constants $\Phi\left(\begin{array}{l}l l_{i j}^{\prime} \\ l^{\prime}\end{array}\right)$.

The volume dependence of the phonon frequencies induced by the volume dependence of the force constants is traditionally modeled by the Grüneisen parameter

$$
\gamma_{k j}=-\frac{\partial \ln \nu_{j}(k)}{\partial \ln V}
$$

which is defined for each branch $j$ and each point $k$ in the first Brillouin zone. But since we are interested in determining the free energy of a system, it is convenient to directly parametrize the volume dependence of the free energy itself. This dependence has two sources: the change in entropy due to the change in the phonon frequencies and the elastic energy change due to the expansion of the lattice:

$$
F(T, V)=E^{*}(V)+F_{H}(T, V),
$$

where $E^{*}(V)$ is the energy of a motionless lattice constrained to remain at volume $V$, while $F_{H}(V)$ is the vibrational free energy of a harmonic system constrained to remain at volume $V$. The equilibrium volume $V^{*}(T)$ at temperature $T$ is obtained by minimizing this quantity with respect to $V$. The resulting free energy at temperature $T$ is then given by $F\left[T, V^{*}(T)\right] .^{16}$

Let us consider a particular case that illustrates the effect of temperature on the free energy, at the cost of a few reasonable assumptions. We assume that

- the elastic energy of the motionless lattice is quadratic in volume;

\footnotetext{
${ }^{15}$ Of course, in general, it could be a general strain dependence, if the symmetry of the crystal is sufficiently low.

${ }^{16}$ Formally, the free energy should be determined by a sum over every possible volume: $-k_{B} T \ln \left\{\Sigma_{V} \exp [-\beta F(V)]\right\}$. However, since the volume is a macroscopic quantity, its distribution can be considered a delta function and the sum reduces to a single term: the free energy at the volume that minimizes the free energy.
}

- the high-temperature limit of the free energy can be used.

As shown in Appendix B, in this approximation, the volume expansion $\Delta V$ as a function of temperature takes on a particularly simple form:

$$
\frac{\Delta V}{N}=\frac{3 k_{B} T \bar{\gamma}}{B}
$$

where $\bar{\gamma}$ is an average Grüneisen parameter:

$$
\bar{\gamma}=\frac{1}{3 N} \sum_{m=1}^{3 N} \frac{V}{\nu_{m}} \frac{\partial \nu_{m}}{\partial V}
$$

The resulting temperature dependence of the free energy is given by

$$
\frac{F(T)}{N}=\frac{F\left(T, V_{0}\right)}{N}-\frac{\left(3 k_{B} T \bar{\gamma}\right)^{2}}{2 B\left(V_{0} / N\right)} .
$$

These expressions provide a simple way to account for thermal expansion. They also allow us to estimate the changes in vibrational entropy as a function of temperature that is due to thermal expansion:

$$
\frac{d S_{\mathrm{vib}}}{d T}=\frac{9 k_{B} \bar{\gamma}^{2}}{B\left(V_{0} / N\right)} k_{B} .
$$

In metallic alloys, this quantity is typically of the order of $10^{-4} k_{B} / \mathrm{K}$.

\section{Simulation}

There are two main simulation-based approaches to handling anharmonicity: Monte Carlo (MC) (Binder and Heermann, 1988) and molecular dynamics (Allen and Tildesley, 1987). While both approaches are able to model anharmonicity at any level of accuracy, they suffer from two limitations. First, they are computationally demanding and therefore have, to date, been limited to simple energy models. Second, they are unable to model quantum-mechanical aspects of vibrations and are therefore limited to the high-temperature limit. ${ }^{17}$ There is an interesting and useful complementarity between the quasiharmonic model and simulation techniques (de Fontaine et al., 1998; Morgan, 1998). Quantum effects typically become negligible in the temperature range where strong anharmonic effects, which cannot be modeled accurately within the quasiharmonic framework, become important.

The use of simulation techniques to determine vibrational properties bypasses the coarse graining framework presented in Sec. II.C: Both configurational and vibrational excitations are treated on the same level. When a simple energy model provides a sufficient accuracy, one can calculate thermodynamic properties directly from $\mathrm{MC}$ simulations where both atomic displace-

\footnotetext{
${ }^{17}$ Monte Carlo simulations that include quantum effects are possible for systems containing a small number of particles.
} 
ments and changes in chemical species are allowed during the simulation (Rittner et al., 1994; Silverman et al., 1995).

While a full determination of a phase diagram from simulations has, to our knowledge, not been attempted, both MD (Ravelo et al., 1998) and MC (de Fontaine et al., 1998; Morgan, 1998) have been used to determine differences in vibrational free energy between two phases. Because neither MD nor MC are able to provide free energies directly, a special integration technique needs to be used. The idea is to express a thermodynamic quantity inaccessible to $\mathrm{MC}$ as an integral of a quantity that can be obtained through MC. A simple example is the change free energy $F$ as a function of temperature at constant pressure, which can be derived from the Gibbs-Helmholtz relation

$$
F\left(T_{2}\right)=F\left(T_{1}\right)-\int_{1 / T_{1}}^{1 / T_{2}} E d(1 / T),
$$

where $E$ is the internal energy. Another example is the change in entropy as a function of temperature (at constant pressure) which can be expressed in terms of the heat capacity:

$$
S\left(T_{2}\right)=S\left(T_{1}\right)+\int_{T_{1}}^{T_{2}} \frac{C_{p}}{T} d T .
$$

Often, the most computationally efficient path of integration between two states is not physically meaningful. For instance, one can gradually change the interatomic potentials during the course of the simulation, in order to model a change in the configuration of the alloy, without requiring atoms to jump between lattice sites. This task is achieved by defining an effective Hamiltonian

$$
H_{\lambda}=(1-\lambda) H^{\alpha}+\lambda H^{\beta}
$$

that gradually switches from the Hamiltonian $H_{\alpha}$ associated with phase $\alpha$ to the Hamiltonian $H_{\beta}$ associated with phase $\beta$ as the switching parameter $\lambda$ goes from 0 to 1 . This convenient path of integration permits the calculation of free energy differences between phases at a reasonable computational cost, with the help of the following thermodynamic relation:

$$
F^{\beta}=F^{\alpha}+\int_{0}^{1}\left(\left\langle H_{\beta}\right\rangle_{\lambda}-\left\langle H_{\alpha}\right\rangle_{\lambda}\right) d \lambda,
$$

where $\left\langle H_{\alpha}\right\rangle_{\lambda}$ is the average of the energy calculated using Hamiltonian $H_{\alpha}$ (and similarly for $\left\langle H_{\beta}\right\rangle_{\lambda}$ ).

\section{Energy models}

Force constants and anharmonic contributions are ultimately always derived from an energy model. In this section, we discuss various energy models, from empirical potential models to first-principles techniques, and the error or bias they may introduce in the vibrational properties.

Simple pairwise potentials or functionals (such as the embedded atom method) are computationally efficient so that all vibrational properties can often be determined without any approximations beyond the ones associated with the specific energy model. For this reason, the use of simple energy models has proven to be an invaluable tool to understand trends in vibrational entropies and to test a number of approximations (de Fontaine et al., 1998; Garbulsky, 1996; Garbulsky and Ceder, 1996; Morgan, 1998; Morgan et al., 1998).

Several potential sources of error can arise when using pair potentials or pair functionals. The first one is that vibrational entropy is extremely sensitive to the precise nature of the relaxations that take place in an alloy and a simple energy model may not be able to accurately predict these relaxations. This problem is particularly apparent when considering the wide range of values found in the different calculations of the vibrational entropy change upon disordering of the $\mathrm{Ni}_{3} \mathrm{Al}$ compound (Ackland, 1994; Althoff et al., 1997; Ravelo et al., 1998; van de Walle et al., 1998). But, as shown in Table IV, most of the discrepancies can be explained from differences in the predicted volume change upon disordering.

This is often aggravated by the fact that simple energy models are often not fitted to phonon properties. The problem was noted in Althoff et al. (1997) where the embedded atom potentials used were fitted to various structural energies and elastic constants (Voter and Chen, 1988). The acoustic modes were accurately extrapolated from the fit to the elastic constants, but the phonon frequencies associated with the optical modes were overestimated by about $10 \% .{ }^{18}$ The question of the accuracy of simple energy models clearly merits further attention. In this respect, the fit of simple energy models to the results of $a b$ initio calculations (Silverman et al., 1995; Shaojun et al., 1998) offers a promising way to include vibrational effects.

In oxides, electronic polarization has to be included in order to correctly model both the low-frequency acoustic modes and the high-frequency optical modes. Electronic polarization in oxides can be approximated with the so-called core and shell model (Dick and Overhausser, 1958).

While quantum-mechanical methods are computationally more intensive, they generally provide more accurate force constants. The most obvious error introduced by the common local-density approximation (LDA) is its systematic underprediction of lattice constants which leads to an overestimation of elastic constants and phonon frequencies. This systematic error makes it difficult to compare the absolute values of calculated vibrational properties with experimental measurements. However, for the purpose of calculating phase diagrams, this bias may be less of a concern, be-

\footnotetext{
${ }^{18}$ Most of the bias in the vibrational entropy introduced by this problem should, however, cancel out when taking the difference in vibrational entropy between two phases where the same problem is present.
} 
TABLE IV. Relation between the vibrational entropy change upon disordering and the volume change upon disordering in various theoretical investigations of the $\mathrm{Ni}_{3} \mathrm{Al}$ compounds.

\begin{tabular}{llll}
\hline \hline$T(\mathrm{~K})$ & $S_{v i b}^{o \rightarrow d}$ & $\Delta V^{o \rightarrow d} / V^{o}(\%)$ & Refs. \\
\hline high & 0.29 & $3 \%$ & (Ackland, 1994) \\
1200 & 0.22 & $2 \%$ & (Ravelo et al., 1998) \\
1000 & 0.15 & $2 \%$ & (Althoff et al., 1997, 1998) \\
1000 & 0.11 & $1.6 \%$ & (Morgan, 1998) Foiles-Daw embedded atom \\
& & & method potentials \\
high & 0.00 & $0.5 \%$ & (van de Walle et al., 1998) \\
\hline \hline
\end{tabular}

cause phase stability is determined by differences in free energies, and one would expect a large part of this systematic error to cancel out.

A practical way to alleviate the LDA bias is to perform calculations at a negative pressure such that the calculated equilibrium volume agrees with the experimentally observed volume. As shown in van de Walle and Ceder (1999), a very good estimate of the required negative pressure can be obtained by a concentrationweighted average of the pressure associated with the elemental solids. For the purpose of calculating elastic properties, this approach appears to outperform the most popular alternative to LDA, the generalized gradient approximation (GGA). ${ }^{19}$

\section{Convergence issues}

The basic formalisms presented in Secs. II.A and V.A provide two natural ways to control the tradeoff between accuracy and computational requirements. In the context of alloy theory (Sec. II.A), the range of the effective clusters interactions included in the cluster expansion controls how accurately the configurational dependence of vibrational properties is modeled. In the context of the harmonic (or quasiharmonic) treatment of lattice vibrations (Sec. V.A), the range of the force constants included in the Born-von Kármán model controls the accuracy of the calculated vibrational properties for a given configuration. In principle, any desired accuracy can be reached, given sufficient computing power, by increasing the range of the interactions in both the cluster expansion and the Born-von Kármán models. This section seeks to answer the important question of how far these two ranges of interactions need to be pushed in order to reach the accuracy required in a typical phase diagram calculation.

\section{Short-range force constant}

The evidence that spring models including only shortrange force constants are able to correctly model vibrational quantities comes from various sources.

\footnotetext{
${ }^{19}$ Part of the success of the "negative pressure" LDA is due to the fact that it uses information regarding the true experimental volume instead of being fully $a b$ initio. But the knowledge of one pressure per element is a relatively small amount of information.
}

First- and second-nearest-neighbor spring model are routinely used to fit data obtained from neutronscattering measurement of phonon dispersion curves (Fultz, Anthony, Nagel, et al., 1995; Nagel et al., 1995). In the theoretical literature, there have been direct studies of the convergence as a function of the range of interaction considered. All $a b$ initio studies find that shortrange force constants (first- or second-nearest neighbor) permit an accurate determination of thermodynamical quantities in metals (van de Walle et al., 1998; van de Walle and Ceder, 2000) and group-IV semiconductors (Garbulsky, 1996). It is important to note that this rapid convergence of most thermodynamic quantities occurs even when the pointwise convergence rate of the phonon DOS is slow. As noted before, this property arises from the fact that thermodynamic quantities are averages taken over all phonon modes and errors tend to average out.

In ionic systems, the presence of long-range electrostatic interactions may require long-range force constants. However, this electrostatic effect can easily be modeled using pair interactions at a moderate computational cost. Once the forces predicted from a simple electrostatic model have been subtracted, the residual forces should be parametrizable with a short-range spring model.

Some of the $a b$ initio studies of convergence have suggested additional simplifications to force-constant tensors (Garbulsky, 1996; van de Walle and Ceder, 2000): instead of attempting to compute all force constants in each tensor, is it possible to obtain reliable results by keeping only the largest terms. We now present a hierarchy of approximations that is a formalization of these findings.

To obtain a more intuitive representation of a given force-constant tensor $\Phi_{\alpha \beta}(i, j)$, we express it in a basis such that the first Cartesian axis is aligned along the line joining atom $i$ and $j$. The second axis is then taken along the highest symmetry direction orthogonal to the first axis while the third axis is chosen to obtain a righthanded orthogonal coordinate system.

In the absence of symmetry, the most general force constant tensor has nine independent elements. The first simplification is to neglect the three-body terms in the harmonic model of the energy (e.g., $\left[x_{\alpha}(i)\right.$ $\left.-x_{\alpha}(j)\right]\left[x_{\beta}(i)-x_{\beta}(k)\right]$ with $\left.\alpha \neq \beta\right)$. Physically, such terms arise from the deformation of the electronic cloud surrounding atom $i$ that is caused by moving atom $j$ and 
that affect the force acting on atom $k$. Clearly, for any force constant other than the nearest neighbor, this effect is negligibly small. Even for nearest-neighbor tensors, it is the most natural contribution to neglect first. In can be readily shown that for a solid consisting only of pairwise harmonic interaction, the tensor associated with a pair of atoms is symmetric:

$$
\Phi_{\alpha \beta}(i, j)=\Phi_{\beta \alpha}(i, j) .
$$

[This constraint is distinct from the conventional constraint: $\Phi_{\alpha \beta}(i, j)=\Phi_{\beta \alpha}(j, i)$.]

The elements of the force-constant tensor can be ranked in decreasing order of expected magnitude based on three simple assumptions:

(1) Force constants associated with stretching a bond are larger than the ones associated with bending it.

(2) Terms relating orthogonal forces and displacements are smaller than those relating parallel forces and displacements.

(3) In the plane perpendicular to the bond, the anisotropy in the force constants is smaller than the magnitude of the force constants themselves.

We then obtain

$$
\Phi(i, j)=\left(\begin{array}{ccc}
a & d+e & d-e \\
d+e & b+c & f \\
d-e & f & b-c
\end{array}\right)
$$

with

$$
|a|>|b|>|c|>|d|>|e|>|f| \text {. }
$$

This hierarchy of force constants is important to keep in mind, given that the off-diagonal elements of the spring tensors are the most difficult to obtain from supercell calculations, requiring much bigger supercells than diagonal elements. There is evidence (van de Walle and Ceder, 2000) that even keeping only the stretching $(a)$ and isotropic bending $(b)$ terms of the nearest-neighbor spring tensor can provide vibrational entropies with an accuracy of about $0.03 k_{B}$. If this observation turns out to be generally applicable, this offers a simple way to account for vibrational effects in phase diagram calculations.

\section{Short-range effective cluster interactions}

If a cluster expansion of the vibrational free energy only requires a small number of effective cluster interactions to accurately model the configurational dependence of the vibrational free energy, it then becomes practical to determine the values of these effective cluster interactions from a small number of very accurate calculations of the vibrational free energy of a few structures.

The issue of the speed of convergence of the cluster expansion is also related to the task of devising efficient ways to compute vibrational properties of disordered alloys: The faster the cluster expansion converges, the easier it is to model a disordered phase (see Appendix C). The calculations of the vibrational entropy change upon disordering has proven to be a very effective way to assess the importance of lattice vibrations (Althoff et al., 1997; Ravelo et al., 1998; van de Walle et al., 1998; van de Walle and Ceder, 2000), since this quantity can be straightforwardly used to estimate the effect of lattice vibrations on transition temperatures with the help of Eq. (4).

The central question is thus whether the cluster expansion of the vibrational free energy converges quickly with respect to the number of effective cluster interactions. This is a question distinct from the range of force constants needed to obtain accurate vibrational properties. The range of effective cluster interactions needed to represent the configurational dependence of vibrational free energy may very well exceed the range of the force constants. Even in simple Born-von Kármán model systems, there is no direct correspondence between effective cluster interactions and force constants, except in special cases (see Sec. VII.A). Once relaxations are introduced in the model, then all hope of a simple correspondence is lost (Morgan et al., 1998).

In this context, the question of the existence of a rapidly converging cluster expansion of vibrational properties has to be answered through numerical experiments. Simple energy models offer the possibility to test, at a reasonable computational cost, the speed of convergence of a cluster expansion. Explicit calculations of a well converged cluster expansion of vibrational entropy in a Lennard-Jones solid (Garbulsky and Ceder, 1996) have indicated that a small number of effective cluster interactions (9) can provide a good accuracy $\left( \pm 0.03 k_{B}\right)$. Other benchmarks of the speed of convergence, based on studies of disordered alloys (de Fontaine et al., 1998; Morgan, 1998; Morgan et al., 1998, 2000), also indicate that concise and accurate cluster expansions are possible. Experiments that seek to link features of projected phonon DOS to the local chemical environment of the atoms (Fultz et al., 1998) suggest that short-range effective cluster interactions should be able to successfully model vibrational entropy differences. One potential source of concern is the difficulty associated with accounting for the size mismatch effect using a short-range effective cluster interaction (Morgan et al., 1998, 2000; van de Walle and Ceder, 2000). In the context of cluster expansions of the energy, relaxations of the atoms away from their ideal lattice site as a result of size mismatch are known to introduce both nonnegligible long-range pair effective cluster interactions and numerous multiplet effective cluster interactions. A cluster expansion of the vibrational free energy is expected to exhibit the same problems.

All the full phonon DOS ab initio calculations of vibrational entropies in alloy systems performed so far have relied on the rapid convergence of the cluster expansion (Garbulsky, 1996; Ozolinsš et al., 1998c; Tepesch et al., 1996; van de Walle et al., 1998; van de Walle and Ceder, 2000). While efforts to quantify the error introduced by truncating the cluster expansion in ab initio 
calculations have been made (Garbulsky, 1996; van de Walle et al., 1998; van de Walle and Ceder, 2000), the issue of the speed of convergence of the cluster expansion in the context of vibrational properties clearly merits further study, especially in light of the importance of the size mismatch effect (van de Walle and Ceder, 2000).

\section{EXPERIMENTAL TECHNIQUES}

The experimental literature on the thermodynamics of lattice vibrations in alloys relies on mainly three techniques. In differential calorimetry measurements, the heat capacity of two samples in a different state of order is compared over a range of temperatures. If the upper limit of the range of temperatures is chosen to be sufficiently low, substitutional exchanges will not occur and the difference in heat capacity can be assumed to arise solely from vibrational effects. Integration of the difference in heat capacity (divided by temperature) then yields a direct measure of the vibrational entropy differences between the two samples of the range of temperature considered. This, of course, assumes that the lower temperature bound is sufficiently low, so that the vibrational entropy of both samples can be assumed to be zero at that temperature. It also assumes that the electronic contribution to the heat capacity is negligible. In practice, both assumptions are typically satisfied. The main problem with this method is that one is usually interested in vibrational entropy differences at the transition temperature of the alloy, which is usually above the upper limit of the temperature range used in the heat-capacity measurements. The heat capacity therefore needs to be extrapolated to high temperature. This constitutes the main source of inaccuracies in this method. Examples of the use of this method can be found in Anthony et al. (1993, 1994), Nagel, Anthony, et al. (1997), Nagel et al. (1995, 1996), and Nagel, Fultz, and Robertson (1997).

A second method is the measurement of phonon dispersion curve through inelastic neutron scattering. For ordered alloys that can be produced in large single crystals, this method is very powerful. Once the dispersion curves along special directions in reciprocal space are measured, they can be used to fit Born-von Kármán spring models which, in turn, yield the normal frequencies for any point in the Brillouin zone. With the help of the standard statistical-mechanics techniques described in Sec. V.A, this information is sufficient to determine the vibrational entropy. Examples of applications of this method can be found in Anthony et al. (1994), Nagel, Anthony, et al. (1997), Fultz, Anthony, Nagel, et al. (1995), Fultz, Anthony, Robertson, et al. (1995), and Nagel et al. (1995). The applicability of this method is unfortunately limited by the availability of large single crystals. The case of disordered alloys presents an even more fundamental problem: Disordered alloys do not have well-defined dispersion curves and there is no straightforward way to fit the spring constants of a spring model from the experimental data. This problem is usually addressed by using the virtual crystal approxi- mation, in which different constituent atoms are replaced by one "average" type of atom (see Appendix C). Unfortunately, this approximation has repeatedly been shown to have a very limited accuracy for the purpose of measuring vibrational entropy differences (Althoff et al., 1997; Nagel et al., 1997; Ravelo et al., 1998; Shaojun et al., 1998). Nevertheless, single-crystal phonon dispersion curve measurements for ordered alloys present a unique opportunity to perform a stringent test of the accuracy of theoretical models.

A third method is the determination of the phonon density of states from incoherent neutron-scattering measurements. In contrast to the preceding approach, this method can readily be applied to disordered systems and to compounds for which single crystals are not available (Bogdanoff et al., 1999; Fultz, Anthony, Nagel, et al., 1995; Nagel et al., 1996; Nagel, Fultz, and Robertson, 1997; Robertson et al., 1999). The main limitation of this approach is that different atomic species have different neutron-scattering cross sections. The scattered intensity at each frequency measures a "density of states," where each mode is weighted by the scattering intensity of the atoms participating in the mode in question. Thus one needs some prior information about the vibrational modes in order to reconstruct the true phonon DOS from the experimental data. In the case of alloys, there is not a one-to-one correspondence between the measured data and the vibrational entropy. This problem can be alleviated by choosing alloy systems where the scattering intensity of each species is similar (Nagel, Fultz, and Robertson, 1997; Robertson et al., 1999).

Other techniques have been used to measure vibrational entropy differences. Some researchers have used the fact that vibrational entropy and thermal expansion are directly related, to estimate vibrational entropy differences from accurate thermal-expansion measurements (Mukherjee et al., 1996, 1998). The measurement of inelastic nuclear resonant scattering spectrum has also been used to relate changes in the phonon DOS to changes in the short-range order of a disordered alloy (Fultz et al., 1998). Finally, relatively noisy estimates of vibrational entropy differences can be obtained from $\mathrm{x}$-ray Debye-Waller factors or from the measurement of mean-square relative displacement (MSRD) of the atoms relative to their neighbors through extended electron energy-loss fine structure (EXELFS) (Anthony et al., 1993).

\section{MODELS OF LATTICE VIBRATIONS}

While the ability to control the level of approximation discussed in the previous section is extremely useful, there remains the problem that, very often, only considering the first few levels in this hierarchy of approximations already involves substantial computational requirements. For this reason, models of lattice vibrations that involve fewer parameters but more physical intuition may provide a practical mean of including vibrational effects in phase diagram calculations. In this section, we 
will present the advantages and weaknesses of each method, in light of the three fundamental mechanisms described in the Sec. IV.

\section{A. The "bond proportion" model}

There have been many attempts [see, for instance, Dyson (1953), Wojtowicz and Kirkwood (1960), Bakker (1982a), Garbulsky and Ceder (1994)] to find ways to express the relationship between the vibrational free energy and the dynamical matrix in a form that illustrates the intuition behind the bond proportion mechanism. In a variety of simple model systems, a convenient exact expression can be derived for the nearest-neighbor effective cluster interaction in the expansion of the vibrational free energy in the high-temperature limit. For simple nearest-neighbor spring models with central forces in linear chains (Bakker, 1982a; Garbulsky and Ceder, 1994; Matthew et al., 1983), square lattices (Bakker, 1982a), or simple cubic lattices (Waegmaekers and Bakker, 1984), the nearest-neighbor effective cluster interaction is given by

$$
V_{1 n n}=\frac{d}{8} k_{B} T \ln \left(\frac{k_{A A} k_{B B}}{k_{A B}^{2}}\right) .
$$

where $k_{A A}, k_{B B}$, and $k_{A B}$ are, respectively, the spring constants associated with $A-A, B-B$, and $A-B$ bonds and $d$ is the dimensionality of the system. It has been noted, on the basis of numerical experiments, that the same expression performs well for other lattices (Garbulsky and Ceder, 1994). This success arises from the fact that, as shown in Appendix E, Eq. (37) is the firstorder approximation to the true vibrational entropy change in a large class of systems which satisfies the following assumptions:

- the high-temperature limit of the vibrational entropy is appropriate;

- the nearest-neighbor force constants can be written as $\Phi(i, j)=k_{\sigma_{i} \sigma_{j}} \phi(i, j)$ where $k_{\sigma_{i} \sigma_{j}}$ denotes the (scalar) stiffness of the spring connecting sites $i$ and $j$ with occupations $\sigma_{i}$ and $\sigma_{j}$ while the $\phi(i, j)$ are dimensionless spring constant tensors. The $\phi(i, j)$ are assumed equivalent under a symmetry operation of the space group of the parent lattice;

- all force constants $k_{\sigma_{i} \sigma_{j}}$ are such that

$$
\left|\frac{k_{\sigma_{i} \sigma_{j}}}{\sqrt{k_{\sigma_{i} \sigma_{i}} k_{\sigma_{j} \sigma_{j}}}}-1\right| \ll 1 .
$$

Equation (37) applies to simple harmonic models with nearest-neighbor springs on the fcc, bcc, or sc primitive lattices (and, approximately, on the hcp lattice), as long as the above assumptions are satisfied. Both stretching and bending terms are allowed in the spring tensors, as long as their relative magnitude is independent of $k_{\sigma_{i} \sigma_{j}}$ (e.g., when the bending terms are always, say, $10 \%$ of the corresponding stretching term, regardless of the magnitude of the stretching term). If force constants, other than bending or stretching terms, are important, the bond proportion model ceases to be valid. This can be seen by the following argument. The bond proportion picture requires every bond of a certain type (for instance, $A-A$ bonds) to have an identical spring tensor. However, the point symmetry of each bond can be different and similar chemical bonds in different environment face different symmetry induced constraints on their spring tensors (Sluiter et al., 1999). The only way to reconcile these observations is use a spring tensor that is compatible with the highest possible symmetry, ensuring that it is also compatible with any other environment with a lower symmetry. With the highest possible symmetry, only two independent terms remain in the spring tensor: the stretching and bending terms.

Equation (37) embodies the essential intuition behind the effect of the alloy's state of order on its vibrational free energy. When one replaces an $A-A$ bond and a $B-B$ bond by two $A-B$ bonds, the vibrational free energy will decrease only if the stiffness of $A-B$ bonds, $k_{A B}$, exceeds the geometrical average stiffness of the bonds between identical species $\sqrt{k_{A A} k_{B B}}$. This observation allows the determination of the expected effect of vibrations on the shape of the phase diagram by simple arguments. The link between the nearest-neighbor effective cluster interaction of the expansion of the vibrational entropy can be summarized by the expression (Garbulsky and Ceder, 1996)

$$
\frac{T_{c}^{\text {config }+ \text { vib }}}{T_{c}^{\text {config }}}=\frac{1}{1 \mp \alpha V_{1 n n} / k_{B} T},
$$

where the "-" and "+" correspond to ordering and segregating systems, respectively, and where $\alpha$ is a dimensionless parameter that only depends on the lattice type and the ordering tendency of the system (for instance, for fcc, $\alpha=1.7$ in ordering systems and $\alpha=9.8$ in segregating systems, while for bcc, $\alpha=6.5$ in both cases).

It is straightforward to include vibrational effects in phase diagram calculations using the bond proportion model. All that is needed is an estimate of the stiffness of $A-A, B-B$, and $A-B$ bonds, which could come, for instance, from supercell calculations of the nearestneighbor force constants in a few simple structures or from the bulk moduli of the pure elements and one ordered compound. The nearest-neighbor effective cluster interaction then obtained can be simply added to the cluster expansion of the energy.

While Eq. (37) is useful to estimate the importance of the bond proportion mechanism in a given system, one can avoid some of the approximations involved in deriving Eq. (37) at the expense of only a modest amount of additional effort. One can find the exact phonon DOS of the nearest-neighbor Born-von Kármán model for a variety of configurations of the alloy, which allows a more accurate cluster expansion of the vibrational energy to be derived. In this fashion, the condition specified in Eq. (38) is no longer needed and the vibrational entropy can be calculated at any temperature. 
It is important to keep in mind that two important assumptions are made when invoking the bond proportion mechanism. First, vibrational entropies are solely determined by the nearest-neighbor force constants. There is theoretical evidence that nearest-neighbor spring models can predict vibrational entropy differences with an accuracy of about $0.02 k_{B}$ in metallic (van de Walle et al., 1998; van de Walle and Ceder, 2000) and semiconductor (Garbulsky, 1996) systems. Given that configurational entropy differences are typically of the order of $0.2 k_{B}$, this precision should be sufficient for practical phase diagram calculations.

The second assumption is that each type of chemical bond is assumed to have an intrinsic stiffness that is independent of its environment. First-principles calculations on the Li-Al (Sluiter et al., 1999) and on the Pd-V system (van de Walle and Ceder, 2000) unfortunately indicate that the stiffness of a chemical bond does change substantially as a function of its environment. This problem is serious, as it considerably limits the applicability of the bond proportion model. These changes of the intrinsic stiffness of the bonds as a function of their environment are precisely the focus of the two other suggested sources of vibrational entropy changes. In summary, while the bond proportion model gives an elegant description of one of the mechanisms suggested to be at the origin of vibrational entropy differences, it completely ignores the two other mechanisms, namely, the volume and size mismatch effects.

\section{B. The Debye model}

Perhaps the most widespread approximation to the phonon DOS $g(\nu)$ is the Debye model [see, for instance, Grimvall (1986) and Ashcroft and Mermin (1976)], where the phonon problem is solved in the acoustic limit. In this case, the phonon DOS is approximated by

$$
g(\nu)=\left\{\begin{array}{l}
\frac{9 \nu^{2}}{\nu_{D}^{3}} \text { if } \nu \leqslant \nu_{D} \\
0 \text { if } \nu>\nu_{D},
\end{array}\right.
$$

where $\nu_{D}=k_{B} \Theta_{D} / h$ and $\Theta_{D}$ is the Debye temperature, given by

$$
\Theta_{D}=\frac{h}{k_{B}}\left(\frac{3 N}{4 \pi V}\right)^{1 / 3} C_{D}
$$

where $C_{D}$ is the Debye sound velocity, defined by

$$
\frac{3}{C_{D}^{3}}=\left\langle\sum_{\lambda=1}^{3} \frac{1}{C_{\lambda}^{3}}\right\rangle,
$$

where the right-hand side is the directional average of a function of the three sound velocities $C_{\lambda}$ (Grimvall, 1986). The free energy of a Debye solid is given by

$$
\begin{aligned}
\frac{F}{N}= & \frac{E^{*}}{N}+\frac{9}{8} k_{B} \Theta_{D} \\
& -k_{B} T\left\{D\left(\frac{\Theta_{D}}{T}\right)-3 \ln \left[1-\exp \left(-\frac{\Theta_{D}}{T}\right)\right]\right\} \\
\approx & \frac{E^{*}}{N}+3 k_{B} T \ln \left(\frac{k \Theta_{D}}{h}\right)
\end{aligned}
$$

in the high-temperature limit, where the Debye function $D(u)$ is given by

$$
D(u)=3 u^{3} \int_{0}^{u} \frac{x^{4} e^{x}}{\left(e^{x}-1\right)^{2}} d x .
$$

Since the Debye sound velocity $C_{D}$ is a complicated function of all elastic constants of the material, an approximation to the Debye temperature that only involves the bulk modulus proves extremely useful. Such an approximation was derived by Moruzzi, Janak, and Schwarz (MJS) (Moruzzi et al., 1988) for cubic materials: ${ }^{20}$

$$
\Theta_{D}=0.617\left(\frac{3}{4 \pi}\right)^{1 / 3} \frac{h}{k_{B}}\left(\frac{\Omega^{1 / 3} B}{\bar{M}}\right)^{1 / 2},
$$

where $\Omega$ is the average atomic volume, $B$ is the bulk modulus, and $\bar{M}$ is the concentration weighted arithmetic mean of the atomic masses. As noted in Garbulsky and Ceder (1994), in the high-temperature limit, the MJS model does not exhibit the required property that the masses have no effect on the vibrational free energy of formation, although using a geometric average of the masses (Garbulsky and Ceder, 1996) fixes this problem.

The quasiharmonic approximation can be used, within Debye theory, to account for mild anharmonicity. In the so-called Debye-Grüneisen approximation, the volume dependence of the phonon DOS is modeled by a single Grüneisen parameter and the effect of volume can be summarized by simply making the Debye temperature volume dependent:

$$
\Theta_{D}=\Theta_{D, 0}\left(\frac{V_{0}}{V}\right)^{\gamma},
$$

where $\Theta_{D, 0}$ is the Debye temperature at volume $V_{0}$ and $\gamma$ is the Grüneisen parameter.

Despite their inaccurate description of the true phonon DOS at high frequencies, the Debye and DebyeGrüneisen models are quite successful at modeling the changes in vibrational properties of a given compound as a function of temperature. For instance, the thermal properties of pure metals (Moruzzi et al., 1988) calculated in the Moruzzi-Janak-Schwarz approximation are surprisingly accurate. The reason for this success is that most thermodynamic quantities (e.g., free energy, en-

\footnotetext{
${ }^{20}$ This approximation has also been used for materials with a lower symmetry (Sanchez et al., 1991; Asta et al., 1993).
} 
tropy, heat capacity, etc.) exhibit their most dramatic variations at low temperature, where the low-frequency phonon modes that are correctly described by the Debye model have a dominant effect. In the hightemperature regime, thermodynamic quantities are determined by the classical equipartition theorem, and any harmonic model gives the correct behavior.

Debye-like models are expected to perform well in systems where the differences in vibrational free energy between compounds can be explained by uniform shifts of the phonon DOS, such as when the volume effect operates alone. Such a behavior has been observed in embedded atom calculations on the Ni-Al system (Ackland 1994; Althoff et al., 1997; Ravelo et al., 1998) but in no other systems so far. The Moruzzi-Janak-Schwarz approximation has been used to include vibrational effects in phase diagram calculations and has resulted in an improved agreement with experimental results (Asta et al., 1993; Colinet et al., 1994; Sanchez et al., 1991).

However, as shown in Garbulsky and Ceder (1996), the Debye approximation and its successors can have significant shortcomings when used to calculate phase diagrams. A significant part of the vibrational freeenergy differences between different compounds arises from changes in the high-frequency portion of the phonon DOS, which Debye-like models describe incorrectly. In some cases, the MJS approximation can even lead to an incorrect prediction of the sign of the vibrational entropy difference (Ozolinšs et al., 1998c; van de Walle and Ceder, 2000).

In summary, the Debye model and its derivatives capture the essential physics behind only one of the advocated mechanisms responsible for the configurational dependence of vibrational free energy: the volume effect. Approximations based on the Debye model, however, fail to account for the possibility that the state of order also has a direct impact (i.e., not through the volume) on the shape of the phonon DOS (as predicted, for instance, by the bond proportion model).

\section{The Einstein model}

The perfect complement to the Debye model is the Einstein model [see, for instance, Ashcroft and Mermin (1976) and McQuarrie (1973)], which focuses on the high-frequency region of the phonon DOS, instead of its low-frequency region. The Einstein model assumes that a crystalline solid can be modeled by a collection of $3 N$ independent harmonic oscillators (three per atom) sharing a common frequency. This frequency can, for instance, be determined by computing the natural frequency of oscillation of one atom when all others are frozen in place. This approach, known as the local harmonic model (LeSar et al., 1989; Sutton, 1989), has proven especially useful to calculate vibrational entropies associated with defects (Najafabadi et al., 1991; Sutton, 1989; Zhao et al., 1993). The Einstein model can also be combined with a Debye model to better fit experimental calorimetry data (Anthony et al., 1994) or thermal-expansion data (Mukherjee et al., 1996).
The local harmonic model is of little use whenever the system of interest exhibits translational symmetry, because the calculations required to determine the unknown parameters of an Einstein model from first principles directly provide force constants. The latter could be used to obtain a more precise description of the DOS rather than the single-value DOS characterizing the Einstein model.

The Einstein model is nevertheless extremely useful for conceptual purposes, as we will now illustrate. As shown in Appendix D, the vibrational free energy of a system is bounded by above and by below by the free energy of two Einstein-like systems:

$$
\begin{aligned}
& \frac{k_{B} T}{2} \ln \left(\frac{h}{k_{B} T} \prod_{i} M_{i}\left[\left(\Phi^{-1}\right)_{i i}\right]^{-1}\right) \\
& \leqslant F_{\mathrm{vib}} \leqslant \frac{k_{B} T}{2} \ln \left(\frac{h}{k_{B} T} \prod_{i} M_{i} \Phi_{i i}\right) .
\end{aligned}
$$

While the upper bound is obtained from the usual local harmonic model, where surrounding atoms do not relax, the lower bound is obtained when the surrounding atoms are allowed to relax freely. Another way to interpret these bounds is that at one extreme, each atom sees the others as having an infinite mass, while at the other extreme, each atom sees the other atoms as being massless. This result supports the view that vibrational free energy can be meaningfully considered as a measure of the average stiffness of each atom's local environment.

A more rigorous way of defining the contribution of an atom to the total vibrational free energy is the use of the projected DOS [see, for instance, Morgan et al. (1998)]. This approach does not in any way simplify the calculation of vibrational properties, because the full phonon DOS is needed as an input, but it is a useful way to interpret the experimentally measured or calculated phonon DOS. To obtain the contribution of atom $i$ to the DOS, the idea is to weight each normal mode by the magnitude of the vibration of atom $i$ :

$$
g_{i}(\nu)=\frac{1}{N} \sum_{j}\left|e_{j}(i)\right|^{2} \delta\left(\nu-\nu_{i}\right),
$$

where $e_{j}$ is the eigenvector (normalized to unit length) associated with the mode of frequency $\nu_{j}$. Since extensive thermodynamic properties are linear in the DOS, atom-specific local thermodynamic properties can be readily defined from the projected DOS. Note that, by construction, all the projected DOS sum up to the true phonon DOS and thus, all the local extensive thermodynamic quantities sum up to the corresponding total quantity.

\section{The "bond stiffness vs bond length" approach}

In $a b$ initio calculations, most of the computational burden comes from the calculation of the force-constant tensors. It would thus be extremely helpful if the force constants determined in one structure could be used to 


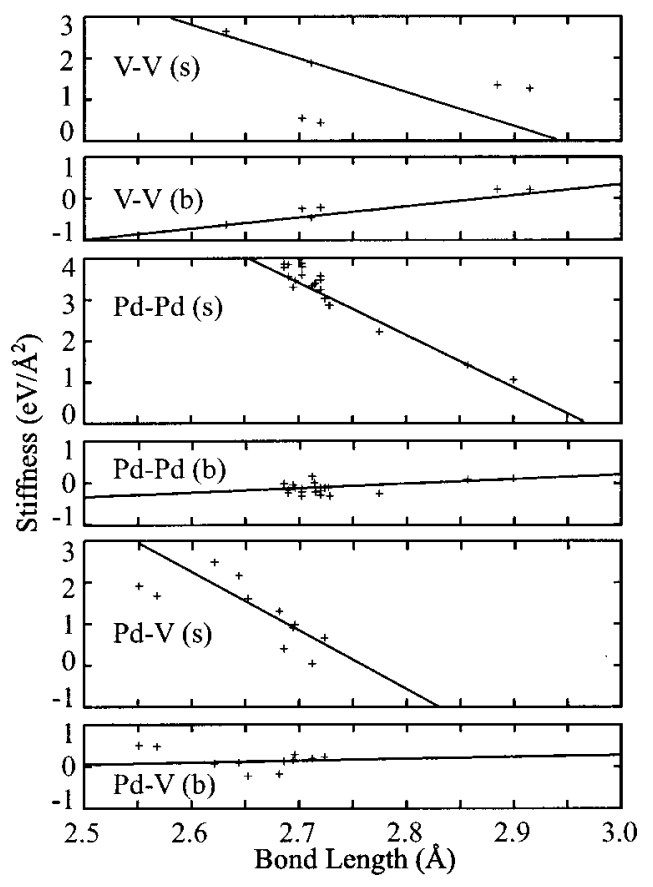

FIG. 4. Stretching (s) and bending (b) terms of the nearestneighbor spring tensor as a function of bond length. Each point corresponds to one type of bond in one of a set of fcc structures $\left(L 1_{2}, D 0_{22}, \mathrm{SQS}-8\right.$, fcc Pd, and fcc V, each taken at two different volumes).

predict force constants in another structure. From the failures of the bond proportion model, however, we know that forces constants obtained from one structure are not directly transferable to another structure (Sluiter et al., 1999; van de Walle and Ceder, 2000)

Nevertheless, a simple modification of the transferable force constant approach yields substantial improvements in precision. First-principles calculation the Pd-V (van de Walle and Ceder, 2000) system revealed that most of the variation in the stiffness of a given chemical bond across different structures can be explained by changes in bond length. ${ }^{21}$ This suggests that the transferable quantity to consider is a bond stiffness vs bond length relationship. As a first approximation, a linear relationship can be used,

$$
\begin{aligned}
& a(l)=a_{0}+a_{1}\left(l-l_{0}\right), \\
& b(l)=b_{0}+b_{1}\left(l-l_{0}\right),
\end{aligned}
$$

where $a$ and $b$ denote the stretching and isotropic bending terms, respectively, and where $a_{0}$ and $b_{0}$ describe the stiffness of the bond at its ideal length $l_{0}$ while $a_{1}$ and $b_{1}$ are analogous to bond-specific Grüneisen parameters. The other parameters of the spring tensor are unlikely

\footnotetext{
${ }^{21}$ The results obtained in the Li-Al system (Sluiter et al., 1999) also suggest that length is a good predictor of stiffness, although these authors did not investigate this matter further.
}
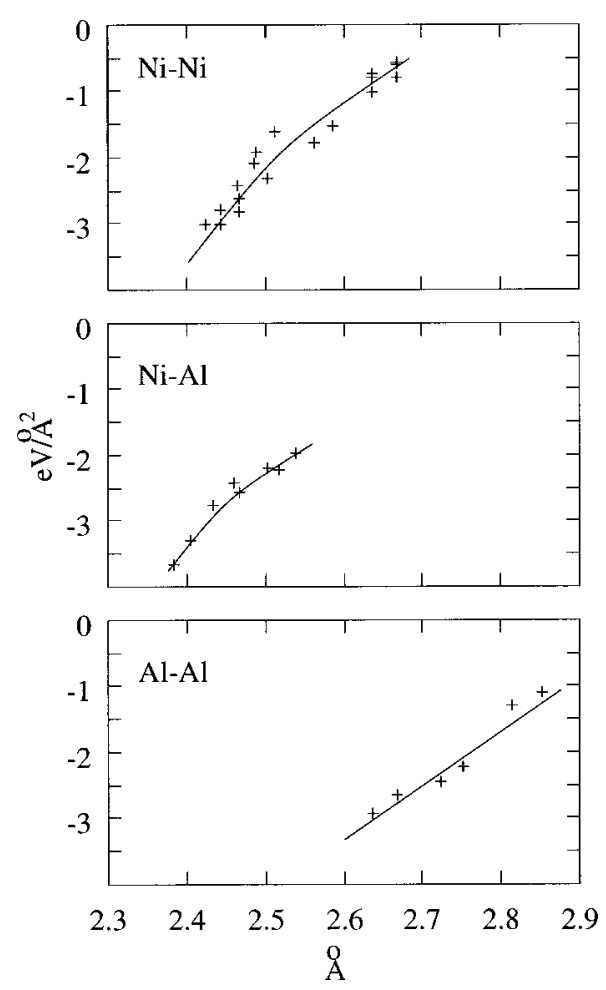

FIG. 5. Bond stiffness as a function of bond length in the $\mathrm{Ni}-\mathrm{Al}$ system. Only stretching terms are shown. Solid lines are the result of a fit to a second-order polynomial. Each point corresponds to one type of bond in one of a set of fcc structures $\left(L 1_{2}, D 0_{22}, \mathrm{SQS}-8\right.$, fcc $\mathrm{Al}$, and fcc Ni, each taken at two different volumes).

to follow such simple relationships because they may be required to vanish according to the local symmetry of the bond, independently of its length (this is discussed in more detail in Appendix E).

This approximation was shown to be successful in the Pd-V system (van de Walle and Ceder, 2000). Figure 4 illustrates the ability of this simple model to predict bond stiffness in different structures. A similar analysis performed with the data on the $\mathrm{Ni}-\mathrm{Al}$ system from van de Walle et al. (1998) is shown in Fig. 5. Table V compares the predictions obtained from the bond stiffness vs bond length model with more accurate calculations.

There are numerous advantages to this approach. From a conceptual point of view, this model presents a concise way to represent all three mechanisms suggested to be the source of vibrational entropy differences. The bond proportion mechanism is the particular case obtained when little changes in bond length occur. The volume effect results from expanding all bonds by the same factor. The size mismatch effect (or the "stiff sphere" picture) is also modeled since the local change in stiffness resulting from locally compressed or expanded regions are explicitly taken into account. A straightforward way to represent the source of vibrational changes is to overlap the stiffness vs length relationship and the changes in average length and stiffness in different states of order, as shown in Fig. 6.

A second advantage of this method is computational 
TABLE V. Comparison between vibrational entropies obtained from the bond stiffness vs bond length model and from a first-nearest-neighbor spring model.

\begin{tabular}{ccc}
\hline \hline $\begin{array}{c}\text { Compound } \\
\text { (structure) }\end{array}$ & $\begin{array}{c}\text { Stiffness vs } \\
\text { length model }\end{array}$ & $\begin{array}{c}1 \mathrm{nn} \\
\text { spring model }\end{array}$ \\
\hline $\mathrm{Pd}_{3} \mathrm{~V} L 1_{2}$ & -4.39 & -4.39 \\
$\mathrm{Pd}_{3} \mathrm{~V} \mathrm{D0} 0_{22}$ & -4.42 & -4.47 \\
$\mathrm{Pd}_{3} \mathrm{~V} \mathrm{SQS}-8$ & -4.56 & -4.54 \\
$\mathrm{Ni}_{3} \mathrm{Al} L 1_{2}$ & -5.57 & -5.55 \\
$\mathrm{Ni}_{3} \mathrm{Al} \mathrm{SQS}-8$ & -5.54 & -5.57 \\
\hline \hline
\end{tabular}

efficiency. The unknown parameters of the model can be determined by a small number of supercell or linear response calculations. After that, the knowledge of the relaxed geometry of a structure is sufficient to determine the stiffness of all chemical bonds. Finding the vibrational entropy of the structure then just reduces to a computationally inexpensive Born-von Kármán phonon problem. It is important to note that the knowledge of the relaxed geometries of a set of structures is a natural by-product of first-principles calculations of structural energies, which are needed to construct the cluster expansion of the energy in phase diagram calculations, whether vibrational effects are included or not. Since computational requirements do not grow rapidly with the number of structures considered, this opens the way for a much more accurate representation of the configurational dependence of the vibrational free energy.

A third advantage of transferable bond stiffness vs bond length relationships is that they contain all the information needed to account for thermal expansion as well, within the quasiharmonic approximation. The slopes of the stiffness vs length relationships for each chemical bond explicitly define the changes in phonon frequencies as volume changes. Since the bulk modulus of each structure is also a by-product of structural energy calculations, all the ingredients needed for a quasi-harmonic treatment of thermal expansion are available.

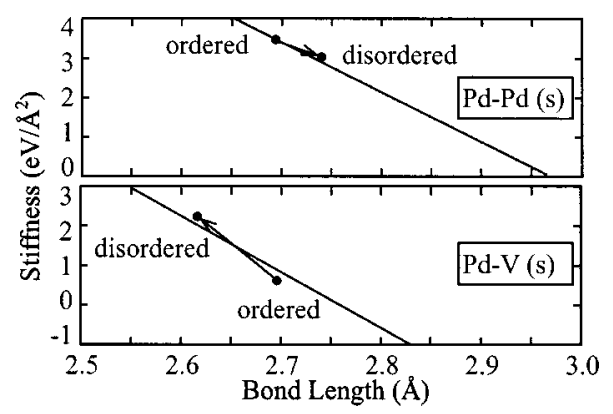

FIG. 6. Shift in average bond stiffness (along the stretching direction) and bond length upon disordering. The fitted line of Fig. 4 is shown for reference.

\section{CONCLUSION}

Lattice vibrations can have a significant impact on phase transition temperatures, short-range order, solubility limits, and the sequence in which phases appear as a function of temperature. The standard framework of alloy theory can be straightforwardly extended to account for lattice vibrations using the concept of coarse graining of the partition function. Once the degrees of freedom associated with lattice vibrations are integrated out, one is left with a standard Ising model, where the energy of each spin configuration is replaced by its vibrational free energy. The efficient evaluation of the vibrational free energy of each configuration is the main problem limiting the inclusion of lattice vibrations in phase diagram calculations. A number of investigations have sought to assess the importance of vibrational effects on phase stability, in order to ensure that the efforts involved in computing vibrational properties are justified. The conclusion of the most reliable of these studies is that vibrational entropy differences are typically on the order of $0.1-0.2 k_{B}$, which is comparable to the magnitude of configurational entropy differences (at most $0.69 k_{B}$ in binary alloys), thereby indicating that vibrations have a non-negligible impact.

The calculation of the vibrational free energy of a particular configuration of the alloy reduces to the wellknown phonon problem in crystals. While the standard harmonic treatment of this problem lacks the ability to model thermal expansion, which can have a significant impact on thermodynamic properties in alloys, this limitation is easily overcome with the help of the quasiharmonic model. An exact solution to the phonon problem for all possible configurations requires excessive computing power. However, the tradeoff between accuracy and computational requirements can be controlled in two ways, namely, through the selection of the range of force constants in the Born-von Kármán model, and through a choice of the number of effective cluster interactions used to expand the configuration dependence of the vibrational free energy. While there is evidence that the range of force constants can be kept very small (first-nearest-neighbor springs), the configurational dependence of the vibrational free energy is too complex to permit a drastic reduction in the number of effective cluster interactions.

Given the substantial computing power required to undertake lattice dynamics calculations, many attempts have been made to devise simpler models. For many years, the Moruzzi-Janak-Schwarz approximation appeared to be a very promising way to include vibrational effects in phase diagram calculations, because it systematically improved the agreement between first-principles calculations and experimental measurements. This success may have been the result of two fortunate circumstances: (i) first-principles phase diagram calculations typically overestimate transitions temperature, and (ii) the Moruzzi-Janak-Schwarz approximation nearly always yields a downward correction to the transition temperature. As the accuracy of phase diagram calculations 
improved through the use of longer-range cluster expansions (Ozolinšs et al., 1998a; Tepesch et al., 1996; van der Ven et al., 1998), the systematic bias in the calculated transitions temperature substantially decreased. Simultaneously, more sophisticated models of lattice vibrations indicated that lattice vibrations do not always result in a reduction in the transition temperatures (Garbulsky, 1996; Garbulsky and Ceder, 1996; van de Walle and Ceder, 2000). The net effect of these two trends is that, although the accuracy of first-principles calculations has increased over the years, obtaining improved agreement with experiment is now a much more stringent test. As a result, perfectly valid and accurate calculations of vibrational effects sometimes reduce the agreement with experiments (Ozolinšs et al., 1998c; Tepesch et al., 1996). Hence, before one can unambiguously assess the importance of lattice vibrations through a full phase diagram calculation, all potential sources of error have to be carefully controlled, such as the precision of the energy model used and more importantly, the accuracy of the cluster expansion. To date, the most convincing evidence that taking lattice vibrations into account significantly improves agreement with experimental results comes from calculations of the lattice dynamics associated with a specific atomic configuration (e.g., a given compound or an isolated point defect) (Ozolinšs and Asta, 2001; Quong and Lui, 1997; Wolverton and Ozolinšs, 2001). In these settings, most sources of errors are under control and definite answers can be given.

Although the availability of more accurate computational tools has revealed that the trends in vibrational entropy differences between phases is far more complex that anticipated ten years ago (Althoff et al., 1997; Garbulsky and Ceder, 1996; Morgan et al., 2000; Sluiter et al., 1999; van de Walle and Ceder, 2000; Wolverton and Ozolinsš, 2001), a simple picture of the mechanisms at work is now emerging. All the known sources of vibrational entropy differences can be conveniently summarized by the bond stiffness vs bond length model (Morgan et al., 2000; van de Walle and Ceder, 2000). In this picture, each type of chemical bond is characterized by a length-dependent spring constant. Changes in vibrational entropy can originate from both changes in the proportion of each chemical bond and changes in their lengths as a result of local and global relaxations. This model not only provides an intuitive understanding of lattice vibrations in alloys, but also a practical way of including their effects in phase diagram calculations. This stiffness vs length relationship of each type of chemical bond can be inferred from a small number of lattice dynamics calculations. The vibrational properties of any configuration can then be obtained at a very low computational cost from the knowledge of the equilibrium geometry of this configuration, an information that is already a natural by-product of any phase diagram calculation.

Future investigations of the effect of lattice vibrations on phase stability should head toward three main directions.

(1) While reporting error bars is an important part of any experimentalists' work, theorists should devote significantly more effort to quantifying the uncertainties of their calculations. This would make it possible to clearly identify situations where the improved agreement with experimental results following the inclusion of vibrational effects is truly significant or merely the result of fortunate coincidences. It is admittedly difficult to quantify the errors introduced by the energy model (such as the LDA), but standard statistical techniques can clearly be used to quantify any error due to fitting the $a b$ initio data with a simple model.

(2) Given the difficulty of extracting vibrational entropies from experimental data, theorists should undertake the computation of quantities that can be directly measured. For instance, a Born-von Kármán model directly enables the simulation of incoherent neutron-scattering data, while the inverse procedure is a highly nonunique operation. The calculation of thermal-expansion coefficients would also be a very sensitive test.

(3) There have so far been very few accurate phase diagram calculations that include the effect of lattice vibrations. The main limitation remains the determination of a cluster expansion that accurately models the configurational dependence of vibrational free energy. The bond length vs bond stiffness model should prove to be an extremely useful tool in achieving this goal. Although this approximation has been very successful in all systems to which it has been applied, the confirmation of its validity in a wider range of systems is crucial. It would also be interesting to devise a hierachy of increasingly accurate approximations that would include the bond length vs bond stiffness model as a particular case.

\section{ACKNOWLEDGMENTS}

This work was supported by the U.S. Department of Energy, Office of Basic Energy Sciences, under Contract No. DE-F502-96ER 45571. Gerbrand Ceder acknowledges support of Union Minière through a Faculty Development Chair. Axel van de Walle acknowledges support of the National Science Foundation under program DMR-0080766 during his stay at Northwestern University.

\section{APPENDIX A: THE ABSENCE OF MASS EFFECTS IN THE HIGH-TEMPERATURE LIMIT}

This appendix shows that the vibrational entropy of formation is independent of the atomic masses in the high-temperature limit, as several authors (Garbulsky and Ceder, 1996; Grimvall and Rosen, 1983) have noted. In the high-temperature limit, the vibrational entropy is 
determined by the product of the frequencies of all normal modes of vibrations $\nu_{m}$, which can be related to the eigenvalues $\lambda_{m}$ of the $3 N \times 3 N$ dynamical matrix $D$ of the system (up to a constant): ${ }^{22}$

$$
\begin{aligned}
\sum_{m} \ln \left(\nu_{m}\right) & =\ln \left(\prod_{m} \nu_{m}\right) \\
& =\frac{1}{2} \ln \left(\prod_{m} \lambda_{m}\right)+\text { const } \\
& =\frac{1}{2} \ln (\operatorname{det} D)+\text { const. }
\end{aligned}
$$

Using the properties of determinants, we can write

$$
\begin{aligned}
\ln (\operatorname{det} D) & =\ln \left[\operatorname{det}\left(M^{1 / 2} \Phi M^{1 / 2}\right)\right] \\
& =\ln [\operatorname{det}(\Phi) \operatorname{det}(M)] \\
& =\ln \left(\operatorname{det}(\Phi) \prod_{i} M_{i}^{3}\right) \\
& =\ln [\operatorname{det}(\Phi)]+3 \sum_{i} \ln M_{i},
\end{aligned}
$$

where $M$ is the $3 N \times 3 N$ diagonal matrix of all the $N$ atomic masses of the system (each repeated three times) while $\Phi$ is $3 N \times 3 N$ the matrix obtained by regrouping all the $3 \times 3$ force constant tensors $\Phi(i, j)$ in a single matrix [analogously to Eq. (14)]. Now consider the change in the value of $\Sigma_{m} \ln \left(\nu_{m}\right)$ when $N_{A}$ atoms of type $A$ and $N_{B}$ atoms of type $B$ are combined to form an alloy. Let the subscripts $A B, A$, and $B$, respectively, denote the properties of an $A_{\left(N_{A} / N\right)} B_{\left(N_{B} / N\right)}$ alloy, a pure crystal of element $A$, and a pure crystal of element $B$ :

$$
\begin{aligned}
\Delta\left(\sum_{m} \ln \left(\nu_{m}\right)\right)= & \sum_{m} \ln \left(\nu_{m}^{A B}\right)-x_{A} \sum_{m} \ln \left(\nu_{m}^{A}\right)-x_{B} \sum_{m} \ln \left(\nu_{m}^{B}\right) \\
= & \frac{1}{2} \ln \left[\operatorname{det}\left(\Phi^{A B}\right)\right]+\frac{3}{2} N_{A} \ln M_{A}+\frac{3}{2} N_{B} \ln M_{B} \\
& -\frac{1}{2} \ln \left[\operatorname{det}\left(\Phi^{A}\right)\right]-\frac{3}{2} N_{A} \ln M_{A}-\frac{1}{2} \ln \left[\operatorname{det}\left(\Phi^{B}\right)\right]-\frac{3}{2} N_{B} \ln M_{B} \\
= & \frac{1}{2} \ln \left[\operatorname{det}\left(\Phi^{A B}\right)\right]-\frac{1}{2} \ln \left[\operatorname{det}\left(\Phi^{A}\right)\right]-\frac{1}{2} \ln \left[\operatorname{det}\left(\Phi^{B}\right)\right] .
\end{aligned}
$$

All the terms involving masses exactly cancel one another.

\section{APPENDIX B: A SIMPLE MODEL OF ANHARMONICITY}

The material presented in this appendix combines standard results regarding the Grüneisen framework that can be found, for instance, in Grimvall (1986).

Two assumptions are made. First, the elastic energy of the motionless lattice is assumed quadratic in volume:

$$
E^{*}(V)=\frac{B}{2 V_{0}}(\Delta V)^{2}
$$

where $B$ is the bulk modulus, $V_{0}$ the equilibrium volume

\footnotetext{
${ }^{22}$ To simplify the exposition and avoid the problem that the dynamical matrix has three zero eigenvalues associated with the possibility of a rigid translation of the system, we assume that some of the atoms of the system are attached to a fixed point of reference by a weak spring. In the thermodynamic limit, this assumption becomes inconsequential.
}

at $0 \mathrm{~K}$ (ignoring zero-point motion), and $\Delta V=V-V_{0}$. Second, the high-temperature limit of the vibrational free energy is used:

$$
F_{\text {vib }}(T, V)=k_{B} T \sum_{i} \ln \left(\frac{h \nu_{i}}{k_{B} T}\right) .
$$

In this approximation, the volume dependence of $F_{\text {vib }}$ takes on a particularly simple form:

$$
\frac{\partial F_{\mathrm{vib}}(T, V)}{\partial V}=\frac{3 N k_{B} T \bar{\gamma}}{V},
$$

where

$$
\bar{\gamma}=\frac{1}{3 N} \sum_{i=1}^{3 N} \frac{V}{\nu_{i}} \frac{\partial \nu_{i}}{\partial V}
$$

is an average Grüneisen parameter. In the hightemperature limit, an average Grüneisen parameter can easily be defined, because the population of the phonon modes is no longer temperature dependent, and any change in entropy can be unambiguously attributed to shifts in phonon frequencies. At lower temperatures, the changes in phonon population would need to be accounted for as well. 
If we assume that the volume dependence of the vibrational free energy is linear in volume, we have

$$
\begin{aligned}
F(T, V) & =E^{*}(V)+F_{\mathrm{vib}}(T, V) \\
& =E^{*}(V)+F_{\mathrm{vib}}\left(T, V_{0}\right)+\left.\frac{\partial F_{\mathrm{vib}}}{\partial V}\right|_{V=V_{0}} \Delta V \\
& =\frac{B}{2 V_{0}}(\Delta V)^{2}+F_{\mathrm{vib}}\left(T, V_{0}\right)+\frac{3 N k_{B} T \bar{\gamma}}{V_{0}} \Delta V .
\end{aligned}
$$

Minimizing this expression with respect to $\Delta V$ yields

$$
\frac{\Delta V}{N}=\frac{3 k_{B} T \bar{\gamma}}{B}
$$

where $3 k_{B} T \gamma / B$ is the coefficient of volumetric thermal expansion. The resulting temperature dependence of the free energy (for one given configuration $\sigma$ ) is given by

$$
\frac{F(T)}{N}=\frac{F\left(T, V_{0}\right)}{N}-\frac{\left(3 k_{B} T \bar{\gamma}\right)^{2}}{2 B\left(V_{0} / N\right)} .
$$

It is interesting to note that half of the vibrational free energy decrease due to thermal expansion is canceled by the energy increase of the motionless lattice. Hence vibrational entropy differences originating from differences in thermal expansion between phases have, relative to other sources of vibrational entropy changes, half the effect on phase stability.

\section{APPENDIX C: MODELING THE DISORDERED STATE}

Although in phase diagram calculations, the use of the cluster expansion bypasses the problem of directly calculating the vibrational entropy of a disordered phase, there are cases where it is of interest to directly calculate the vibrational properties of the disordered state. For instance, in studies that seek to assess the importance of lattice vibrations (Althoff et al., 1997; Ozolinš et al., 1998c; Ravelo et al., 1998; van de Walle et al., 1998), it is instructive to compute the vibrational entropy change upon disordering an alloy, since this quantity can be straightforwardly used to estimate the effect of lattice vibrations on transition temperatures with the help of Eq. (4). Here are the most common methods used to model the disordered state.

Perhaps the most obvious and brute force approach to modeling the disordered state is the use of a large supercell where the occupation of each site is chosen at random. This approach was chosen in all embedded atom method calculations (Ackland, 1994; Althoff et al., 1997; Morgan et al., 1998; Ravelo et al., 1998) as well as in other investigations (Shaojun et al. 1998). Unfortunately, it is generally not feasible in the case of $a b$ initio calculations.

The virtual crystal approximation (VCA) consists of replacing each atom in a disordered alloy by an "average" atom whose properties are determined by a concentration weighted average of the properties of the constituents. In the limit where the chemical species have nearly identical properties, this approximation is justified. This model has been commonly used to interpret neutron scattering measurements of phonon dispersion curves in the case of disordered alloys (Fultz, Anthony, Nagel, et al., 1995; Nagel et al., 1995). It has also been used in a some theoretical investigations (Cleri and Rosato, 1993; Persson et al., 1999). However, the virtual crystal approximation has been repeatedly shown to be insufficiently accurate for the purpose of calculating differences in vibrational entropies between distinct compounds (Althoff et al., 1997; Nagel et al., 1997; Ravelo et al., 1998; Shaojun et al., 1998). Its weaknesses are numerous: It is unable to model bond proportion effects, volume effects, and local relaxations. It also fails to give a mass-independent high-temperature limit.

Special quasirandom structures (SQS's) (Zunger et al., 1990) combine the idea of cluster expansion with the use of supercells. Special quasirandom structures are the periodic structures that best approximate the disordered state in a unit cell of a given size. The quality of a special quasirandom structure is described by the number of its correlations that match the ones of the true disordered state. There is thus a direct connection between cluster expansions and special quasirandom structures: If there exists a truncated cluster expansion that is able to predict properties of the disordered state there exists a special quasirandom structures that provides an equally accurate description of the disordered state.

Special quasirandom structures have been very successfully used to obtain electronic and thermodynamic properties of disordered materials [see, for example, Hass et al. (1990)]. The accuracy of the special quasirandom structures approach in the context of phonon calculations has been benchmarked using embedded atoms potentials which allow for the comparison with the "exact" vibrational entropy of the disordered state with a large supercell (Morgan et al., 1998). It has been found that, for the purpose of calculating vibrational properties, a special quasirandom structure having only eight atoms in its unit cell already provides a good approximation of the disordered state in the case of an fcc alloy at concentration 3/4. While the performance of this small special quasirandom structures is remarkable in a model system where local relaxations are disallowed, it tends to degrade somewhat when relaxations are allowed to take place. This effect can naturally be explained by the fact that relaxations are known to introduce important multibody terms in the cluster expansion, which translates into the requirement that the special quasirandom structures must correctly reproduce the corresponding multibody correlations.

The success of small special quasirandom structures opened the way for the use of more accurate energy models to calculate vibrational properties of disordered alloys. Special quasirandom structures have been applied to the $a b$ initio calculation of vibrational entropy in disordered $\mathrm{Ni}_{3} \mathrm{Al}$ and $\mathrm{Pd}_{3} \mathrm{~V}$ alloys (van de Walle et al., 1998; van de Walle and Ceder, 2000).

\section{APPENDIX D: THE EINSTEIN MODEL}

In the Einstein model of a solid, the free energy, in the high-temperature limit, is given by 


$$
\begin{aligned}
F & =k_{B} T \ln \left(\frac{h}{k_{B} T} \prod_{i} \nu_{i}\right) \\
& =\frac{k_{B} T}{2} \ln \left(\frac{h}{k_{B} T} \operatorname{det} D\right) \\
& =\frac{k_{B} T}{2} \ln \left(\frac{h}{k_{B} T} \operatorname{det}\left(M^{1 / 2} \Phi M^{1 / 2}\right)\right) \\
& =\frac{k_{B} T}{2} \ln \left(\frac{h}{k_{B} T} \operatorname{det} M \operatorname{det} \Phi\right),
\end{aligned}
$$

where $D$ and $\Phi$ are, respectively, the $3 N \times 3 N$ dynamical matrix and force-constant matrix of the system while $M$ is the matrix of the masses:

$$
M_{i j}=\delta_{i j} M_{j} .
$$

It can be shown (van de Walle, 1995) that for any positive definite matrix $\Phi$,

$$
\operatorname{det} \Phi \leqslant \prod_{i} \Phi_{i i}
$$

implying that

$$
F \leqslant \frac{k_{B} T}{2} \ln \left(\frac{h}{k_{B} T} \prod_{i} M_{i} \Phi_{i i}\right),
$$

where the right-hand side expression is nothing but the free energy of the system in the Einstein approximation. A lower bound can be obtained by a similar technique, by using the inverse of the force-constant matrix

$$
\operatorname{det} \Phi \geqslant\left[\prod_{i}\left(\Phi^{-1}\right)_{i i}\right]^{-1} .
$$

The interpretation of the inverse $\Phi$ is simple: It is the matrix that maps forces $F$ exerted on the atoms to the resulting displacements $u$ of the atoms,

$$
u=\Phi^{-1} F .
$$

While $\Phi_{i i}$ is related to the oscillation frequency of a single atom when all other atoms are held in place, $\left[\left(\Phi^{-1}\right)_{i i}\right]^{-1}$ is related to the oscillation frequency of an atom $i$ when all surrounding atoms are allowed to relax so that the force exerted on them remains zero as atom $i$ moves. Atom $i$ has mass $M_{i}$ while all other atoms are considered massless and relax instantaneously. Atoms located infinitely far away from atom $i$ are held in place with an infinitesimal force.

In conclusion, the free energy of a system is bounded by above and by below by the free energy of two Einstein-like systems:

$$
\begin{aligned}
& \frac{k_{B} T}{2} \ln \left(\frac{h}{k_{B} T} \prod_{i} M_{i}\left[\left(\Phi^{-1}\right)_{i i}\right]^{-1}\right) \\
& \leqslant F \leqslant \frac{k_{B} T}{2} \ln \left(\frac{h}{k_{B} T} \prod_{i} M_{i} \Phi_{i i}\right) .
\end{aligned}
$$

APPENDIX E: DERIVATION OF THE BOND PROPORTION MODEL

This appendix generalizes the results found in Bakker (1982a), Matthew et al. (1983), Garbulsky and Ceder (1994), and Waegmaekers and Bakker (1984) in order to handle more general lattice types. We show that, in an important class of systems, the bond proportion model is in fact the first-order approximation to the true change in vibrational entropy induced by a change in the proportion of the different types of chemical bonds.

The alloy system is assumed to satisfy the following conditions:

- the high-temperature limit is appropriate;

- the nearest-neighbor force constants can be written as $\Phi(i, j)=k_{\sigma_{i} \sigma_{j}} \phi(i, j)$ where $k_{\sigma_{i} \sigma_{j}}$ denotes the (scalar) stiffness of the spring connecting sites $i$ and $j$ with occupations $\sigma_{i}$ and $\sigma_{j}$ while the $\phi(i, j)$ are dimensionless spring constant tensors. The $\phi(i, j)$ are assumed equivalent under a symmetry operation of the space group of the parent lattice;

- all force constants $k_{\sigma_{i} \sigma_{j}}$ are such that

$$
\frac{k_{\sigma_{i} \sigma_{j}}}{\sqrt{k_{\sigma_{i} \sigma_{i}} k_{\sigma_{j} \sigma_{j}}}} \ll 1 .
$$

Consider a $d$-dimensional solid made of $N$ atoms connected by springs characterized by symmetrically equivalent tensors $k \phi(i, j)$. Without loss of generality, the masses of all atoms are set to unity since the formation entropies in the high-temperature limit are independent of the atomic masses (see Appendix A). In the high-temperature limit, the vibrational free energy per atom is given by

$$
F_{\mathrm{vib}}=\frac{k_{B} T}{2 N} \sum_{m} \ln \lambda_{m},
$$

where the sum is taken over the nonzero eigenvalues $\lambda_{m}$ of the dynamical matrix $D$ of the system. (The zero eigenvalues correspond the modes where the whole crystal moves rigidly. In the thermodynamic limit, these few missing degrees of freedom are inconsequential.)

Because all springs in the system are equivalent to each other, matrix $D$ can be written as

$$
D=k C,
$$

where $C$ is a matrix of dimensionless geometrical factors independent of $k$ but specific to the type of lattice. From this expression of $D$, it follows naturally that eigenvectors of $D$ are independent of $k$ and that its eigenvalues can be written as

$$
\lambda_{m}=k l_{m},
$$

where the $l_{m}$ are geometric factors independent of $k$.

Consider what happens to $S_{\text {vib }}$ when the stiffness of one of the springs is changed from $k$ to $k+\Delta k$. Let $\Delta D$ 
denote the corresponding change in matrix $D$. To the first order, the resulting changes in the eigenvalues are given by

$$
\Delta \lambda_{m}=u_{m}^{\prime} \Delta D u_{m},
$$

where $u_{m}$ is the (dimensionless) eigenvector of $D$ associated with eigenvalue $\lambda_{m}$. Since $D$ is linear in the spring constants, we can write

$$
\Delta D=\Delta k B,
$$

where $B$ is matrix of geometrical factors independent of $k$ and $\Delta k$ but specific to the type of lattice. While matrix $B$ also depends on which spring is being modified, the matrices $B$ corresponding to each spring are equivalent under a symmetry operation of the crystal's space group. The changes in the eigenvalues can then be expressed as

$$
\Delta \lambda_{i}=\Delta k u_{m}^{\prime} B u_{m} \equiv \Delta k g_{m},
$$

where $g_{i}$ is a dimensionless number independent of $k$ and $\Delta k$.

Substituting these results into Eq. (E2), we obtain

$$
F_{\mathrm{vib}}=\frac{k_{B} T}{2 N} \sum_{m} \ln \left(k l_{m}+\Delta k g_{m}\right) .
$$

To the first order, we can express the vibrational entropy change as

$$
\begin{aligned}
\Delta F_{\mathrm{vib}} & =\left.\frac{\partial F_{\mathrm{vib}}}{\partial \Delta k}\right|_{\Delta k=0} \Delta k \\
& =\frac{k_{B} T}{2 N} \sum_{m} \frac{g_{m}}{k l_{m}} \Delta k \\
& =k_{B} T\left(\frac{1}{2 N} \sum_{m} \frac{g_{m}}{l_{m}}\right) \frac{\Delta k}{k} \\
& \equiv k_{B} T G \frac{\Delta k}{k},
\end{aligned}
$$

where $G$ is a dimensionless geometrical factor depending only on the lattice type.

In the limit of $\Delta k \ll k$, we can obtain the change in vibrational entropy due to a change in all the spring constants by simply summing the effect of the change $\Delta k_{s}$ in the stiffness of each spring $s$ :

$$
\Delta F_{\mathrm{vib}}=k_{B} T G \sum_{s} \frac{\Delta k_{s}}{k} .
$$

To determine the value of $G$, we consider the following particular case for which the exact vibrational entropy change is known. Once the value of $G$ is known, it can be used in any other case sharing a same lattice type.

In a solid bound by springs of stiffness $k$ is given by, if the stiffness of all springs is increased by $\Delta k$, each eigenvalue $\lambda_{m}$ becomes $\lambda_{m}[(k+\Delta k) / k]$ and the vibrational entropy becomes

$$
\begin{aligned}
F_{\mathrm{vib}}^{\prime} & =\frac{k_{B} T}{2 N} \sum_{i} \ln \left(\lambda_{i} \frac{k+\Delta k}{k}\right) \\
& =\frac{k_{B} T}{2 N} \sum_{i}\left(\ln \lambda_{i}+\ln \frac{k+\Delta k}{k}\right) \\
& =F_{\mathrm{vib}}+\frac{k_{B} T}{2 N} \sum_{i} \ln \frac{k+\Delta k}{k} \\
& \approx F_{\mathrm{vib}}+k_{B} T \frac{N d}{2 N} \frac{\Delta k}{k}+O\left[(\Delta k)^{2}\right] \\
& =F_{\mathrm{vib}}+k_{B} T \frac{N d}{2 N} \frac{1}{Z N / 2} \sum_{s} \frac{\Delta k}{k} \\
& =F_{\mathrm{vib}}+\frac{k_{B} T d}{Z N} \sum_{s} \frac{\Delta k}{k},
\end{aligned}
$$

where $Z$ is the number of nearest neighbors and $\Sigma_{s}$ denotes a sum over all nearest-neighbor bonds. Since this result is exact to the first order, we can compare it to Eq. (E8) and identify the unknown constant $G$ to be $d / Z N$. We thus obtain the following result:

$$
\Delta F_{\mathrm{vib}}=\frac{k_{B} T d}{Z N} \sum_{s} \frac{\Delta k_{s}}{k} .
$$

We now turn to the problem of calculating the vibrational entropy of mixing in a binary alloy. We first define a normalized $N d \times N d$ dynamical matrix $\hat{D}$ as follows:

$$
\hat{D}_{\alpha \beta}(i, j)=\frac{\Phi_{\alpha \beta}(i, j)}{\sqrt{k_{\sigma_{i} \sigma_{i}} k_{\sigma_{j} \sigma_{j}}}},
$$

where $k_{\sigma_{i} \sigma_{i}}$ is the spring constant of an $A$-A bond if site $i$ is occupied by an $A$ atom similarly for a site occupied by a $B$ atom. For the purpose of calculating free energy of formation, this normalized dynamical matrix gives the same result as the usual dynamical matrix because the factors in the denominator exactly cancel out, for the same reason masses cancel out (see Appendix A). This transformation normalizes the spring constant associated with $A-A$ bonds and $B-B$ bonds to 1 while the spring constant associated with $A-B$ bond becomes $\left(k_{A B} / \sqrt{k_{A A} k_{B B}}\right)$ where $k_{A B}, k_{A A}$, and $k_{B B}$, respectively, denote the true spring constants of $A-B, A-A$, and $B-B$ bonds. The usefulness of this normalization is to extend the applicability of Eq. (E9) to the case where $k_{A A}$ and $k_{B B}$ are very different.

Let us start with a phase separated mixture of $A$ and $B$ atoms. Let us think of this system as one where all atoms are identical but where the springs connecting them can be either one of three types $A-A, B-B$, or $A-B$, where the springs are placed defines which type of atom sits at each site. We now replace one $A-A$ bond in the pure $A$ phase by an $A-B$ bond and one $B-B$ bond in the pure $B$ phase by an $A-B$ bond. By Eq. (E9), the resulting change in vibrational entropy per atom is 


$$
\Delta F_{\mathrm{vib}}=\frac{k_{B} T d}{Z N}\left(\frac{k_{A B}}{\sqrt{k_{A A} k_{B B}}}-1+\frac{k_{A B}}{\sqrt{k_{A A} k_{B B}}}-1\right) .
$$

To satisfy the assumptions of the above derivation, we require that $k_{A B} / \sqrt{k_{A A} k_{B B}} \ll 1$. If we create a total number $n_{A B}$ of $A-B$ bonds, we perform the above operation $n_{A B} / 2$ times and the vibrational entropy change is

$$
\Delta F_{\mathrm{vib}}=\frac{n_{A B}}{2} \frac{k_{B} T d}{Z N}\left(\frac{k_{A B}}{\sqrt{k_{A A} k_{B B}}}-1+\frac{k_{A B}}{\sqrt{k_{A A} k_{B B}}}-1\right) .
$$

To the first order (when $k_{A B} / \sqrt{k_{A A} k_{B B}} \ll 1$ ), this expression is equivalent to

$$
\Delta F_{\mathrm{vib}}=\frac{n_{A B}}{N} \frac{k_{B} T d}{2 Z} \ln \left(\frac{k_{A B}^{2}}{k_{A A} k_{B B}}\right) .
$$

The nearest-neighbor effective cluster interaction of the cluster expansion of the vibrational free energy is thus

$$
V_{1 n n}=\frac{d}{8} k_{B} T \ln \left(\frac{k_{A A} k_{B B}}{k_{A B}^{2}}\right) .
$$

\section{APPENDIX F: INSTABILITY}

An extreme case of anharmonicity occurs when the energy surface, in the neighborhood of a configuration $\sigma$, has no local minimum. As noted in Craievich et al. (1997) and Craievich and Sanchez (1997), this situation occurs sufficiently frequently to deserve a particular attention. A typical example of such a situation occurs when the fcc-based $L 1_{0}$ structure is unstable with respect to a deformation along the Bain path, which leads to a bcc-based $B 2$ structure. While it is possible to construct a separate cluster expansion for the fcc and bcc phases, the fundamental question that arises is: What is the free energy of the $L 1_{0}$ structure? Since it is unstable, the standard harmonic expression for the free energy can clearly not be used.

One suggested solution to this problem, described in Craievich and Sanchez (1997), is to perform the coarse graining in a different order than presented in Sec. II.C. The sum over configurations is performed first, and the vibrational properties of the configurational averaged alloy are then calculated. The main limitation of this approach is that it would be extremely difficult to compute the averaged vibrational properties by any other method than by the so-called virtual crystal approximation (see Appendix C). Another limitation is that it only addresses instabilities with respect to cell shape distortions, ignoring instabilities with respect to internal degrees of freedom (i.e., atomic positions).

In this section, we present another approach to solve the instability problem. We argue that the general formalism developed in Sec. II.C can in fact be adapted to allow for instability.
While the coarse graining technique is most naturally interpreted as integrating out the "fast" degrees of freedom (e.g., vibrations) before considering "slower" ones (e.g., configurational changes) (Ceder, 1993), the time scale of the various types of excitations is, in fact, irrelevant. The partition function is simply a sum over states which can be calculated in any order. As long as we can associate any vibrational state $v$ of the system with a configuration $\sigma$, the coarse graining procedure remains valid.

Under this point of view, it is clear that it does not matter whether there is even a local minimum of energy in the portion of phase space associated with configuration $\sigma$. What is important, however, is that the neighborhood of configuration $\sigma$ in phase space is thoroughly sampled (i.e., that the constrained system is ergodic) over a macroscopic time scale. There is no need for ergodicity within the time scale of the configurational excitations. If the neighborhood of a given configuration $\sigma$ is not fully sampled before the alloy jumps to another configuration $\sigma^{\prime}$, it is still possible that the unsampled portion of phase space around $\sigma$ will be visited at a later time, when the system returns to the neighborhood of configuration $\sigma$. The ergodicity requirement at the macroscopic time scale imposes the important but intuitively obvious constraint that the phase space neighborhood of configuration $\sigma$ cannot contain states that are associated to different phases of the system.

This discussion shows that there is no fundamental limitation to the applicability of the standard coarse graining framework in the presence of instability. However, we still need to describe how the free energy of an unstable configuration could be determined in practice. The task is simplified by the fact that the free energy of an unstable state does not need to be extremely accurately determined, because unstable states are relatively rarely visited, even at high temperatures. Nevertheless, it is important to assign a free energy to those unstable states, to ensure that the Ising model used to represent the alloy is well defined.

The free energy associated with one configuration can be obtained by integrating $\exp [-\beta E(\sigma, v)]$ with respect to $v$ over the portion of phase space associated with $\sigma$. In the classical limit, we can label the vibrational states $v$ by the position each particle takes and the integration limits can be found by geometrical arguments. The quantum-mechanical equivalent of this operation is complex, ${ }^{23}$ but unlikely to be needed in practice. The unstable states are essentially never visited at low tem-

\footnotetext{
${ }^{23}$ The quantum partition function can be written as the trace of the matrix $\exp (-\beta H)$, where $H$ is the (multibody) Hamiltonian of the system. The trace can computed in any convenient basis and in particular one could use Dirac delta functions. In this fashion, it is possible to define a localized free energy by summing only over the delta functions located in the neighborhood of one configuration $\sigma$.
} 
peratures, where a quantum-mechanical treatment would be essential. ${ }^{24}$

Focusing on the classical limit, we consider an unstable configuration $\sigma$. Let $D$ be the dynamical matrix evaluated at the saddle point of the energy surface closest to the ideal undistorted configuration $\sigma .^{25}$ We consider that when the state $v$ of the system is such that one atom moves away from its position at the saddle point by more than $r$, it should no longer be considered part of configuration $\sigma$. For an instability with respect to internal degrees of freedom (atomic positions), a natural choice for $r$ would be half the average nearest-neighbor interatomic distance. For an instability with respect to unit cell deformation, $r$ could be half the change in the average nearest-neighbor distance induced by the displacive transformation.

The boundedness of the portion of phase space associated with $\sigma$ can be accounted for by replacing the usual classical partition function associated with one normal mode of oscillation $i$ by

$$
\frac{1}{h} \int_{-L_{i}}^{L_{i}} \exp \left(-\frac{1}{2} \beta \dot{s}^{2}\right) d \dot{s} \int_{-L_{i}}^{L_{i}} \exp \left(-\frac{1}{2} \beta \lambda_{i} s^{2}\right) d s,
$$

where $\lambda_{i}$ is the $i$ th eigenvalue of the dynamical matrix, $h$ is Planck's constant, and $L_{i}$ is a measure of the size of the phase space neighborhood of $\sigma$ along the direction associated with normal mode $i$. This size parameter can be expressed in terms of the parameter $r$ just introduced. Let $u_{i}(j)=e_{i}(j) / \sqrt{M_{j}}$ where $e_{i}$ is the $i$ th eigenvector of $D$ and $M_{j}$ is the mass of atom $j$. After normalizing $u_{i}$ so that $\Sigma_{j} u_{i}^{2}(j)=N$, the number of atoms in the system, we can then write

$$
L_{i}=r\left[\max \left\|u_{i}(j)-u_{i}\left(j^{\prime}\right)\right\|\right]^{-1},
$$

where the maximum is taken over all nearest-neighbor pairs of atoms $j, j^{\prime}$. This choice of integration bounds approximately defines a neighborhood of $\sigma$ such that no atom moves farther than $r$ from its position at the saddle point (relative to its neighbors). In this approximation, the free energy of an unstable state is given by

$$
\frac{F}{N}=\frac{E^{*}}{N}-\frac{k_{B} T}{N} \sum_{i} \ln \left[\frac{k_{B} T}{h \nu_{i}} \operatorname{erf}\left(L_{i} \sqrt{\frac{2\left(\pi \nu_{i}\right)^{2}}{k_{B} T}}\right)\right],
$$

where $\nu_{i}$ is the frequency of normal mode $i$ and where the error function for real or imaginary arguments is given by

\footnotetext{
${ }^{24}$ This observation is related to the fact that quasiharmonic approximation, which allows a quantum-mechanical treatment, is accurate up to a temperature where the classical limit is reached.

${ }^{25}$ It is possible that an unstable configuration $\sigma$ cannot be associated with a saddle point and the derivation would have to be modified. In particular the bounds of integration would have to be made asymmetric.
}

$$
\operatorname{erf}(u)=\frac{2 u}{\sqrt{\pi}} \int_{0}^{1} e^{-u^{2} s^{2}} d s .
$$

The suggested definition of the free energy of an unstable configuration has interesting properties. First, as the neighborhood size $L_{i}$ increases, the expression reduces to the usual harmonic expression. The effect of the correction is not limited to unstable modes: Modes that are so soft that it is likely that the motion of the atoms exceeds $r$ are also affected. There may obviously be other definitions of $L_{i}$. The above example simply gives an example of how it could be calculated.

Going back to our initial example of the $L 1_{0} \rightarrow B 2$ instability, we can now outline how this problem could be handled within the traditional coarse graining scheme. Two separate clusters expansion need to be constructed, one for the bcc phases and one for the fcc phases. But since we now know how to assign a free energy to the unstable $L 1_{0}$ configuration, the fcc cluster expansion can be successfully defined. The free energy attributed to the $L 1_{0}$ configuration should be sufficiently high so that the free energy curve of the fcc phase in the vicinity of 0.5 concentration will lie above the free energy curve of the bcc phase, as it should. The fact that both cluster variation method or Monte Carlo calculations on the fcc lattice would attribute a positive probability to $L 1_{0}$-like structures should not be regarded as a problem: This is precisely what will ensure that the calculated fcc free energy curve lies above the bcc one.

The discussion has so far been concerned with the expression of the partition function, which is the relevant quantity to consider when the phase diagram is calculated with the cluster variation method or the lowtemperature expansion. Let us now consider the implications of this approach to Monte Carlo simulations. Thermodynamic quantities derived from averages, such as the average energy, are obviously unaffected by the presence of unstable configurations. For quantities derived from fluctuations, such as the heat capacity, slight modifications are needed. In traditional Monte Carlo simulations, the heat capacity arising from vibrational degrees of freedom is consistently neglected, and any thermodynamic quantity obtained from Monte Carlo can be unambiguously interpreted as the configurational contribution. In the more general setting presented here, there is an overlap between vibrational and configurational fluctuations and the only way to obtain welldefined thermodynamic quantities is to fully account for the vibrational fluctuations. Fortunately, there is a straightforward way to do so. The total variance of the energy (or any other quantity) can be exactly expressed as a sum of the variance within each configuration $\sigma$ and the variance of the average energy of each configuration:

$$
\begin{aligned}
\left\langle E^{2}\right\rangle-\langle E\rangle^{2}= & \sum_{\sigma} \sum_{v \in \sigma} P_{\sigma v} E_{\sigma v}^{2}-\left(\sum_{\sigma} \sum_{v \in \sigma} P_{\sigma v} E_{\sigma v}\right)^{2} \\
= & {\left[\sum_{\sigma} P_{\sigma} \bar{E}_{\sigma}^{2}-\left(\sum_{\sigma} P_{\sigma} \bar{E}_{\sigma}\right)^{2}\right] } \\
& +\sum_{\sigma} P_{\sigma}\left(\sum_{v \in \sigma} \frac{P_{\sigma v}}{P_{\sigma}}\left(E_{\sigma v}-\bar{E}_{\sigma}\right)^{2}\right)
\end{aligned}
$$


A. van de Walle and G. Ceder: Lattice vibrations and substitutional alloys

where $P_{\sigma v}$ is the probability of finding the system in state $\sigma, v$ while $P_{\sigma}=\Sigma_{v \in \sigma} P_{\sigma v}$ and $\bar{E}_{\sigma}$ $=\Sigma_{v \in \sigma}\left(P_{\sigma v} / P_{\sigma}\right) E_{\sigma v}$. The first term is the usual fluctuation obtained from Monte Carlo. The second term is a correction which takes the form of a simple configuration average of fluctuations within each configuration. The fluctuation of a system constrained to remain in the vicinity of configuration $\sigma$ is usually just as simple to determine as its average properties. In the case of energy, the fluctuations within each configuration are simply related to the heat capacity of a harmonic solid.

The main objective of this section was to show that there is no fundamental problem associated with unstable states in coarse graining formalism. While it is true that the free energy of an unstable configuration is not uniquely defined, once a particular way to coarse grain phase space is chosen, the free energy of all configurations can be defined in a consistent fashion. There are admittedly some practical issues to be resolved regarding the practical implementation of coarse graining in the presence of instabilities, but the approach suggested in this section indicates that these difficulties can be overcome.

\section{REFERENCES}

Abrikosov, I. A., A. V. Ruban, D. Ya Kats, and Y. H. Vekilov, 1993, J. Phys.: Condens. Matter 5, 1271.

Ackland, G. J., 1994, in Alloy Modelling and Design, edited by G. Stocks and P. Turchi (The Minerals, Metals and Materials Society, Pittsburgh, PA), p. 149.

Ackland, G. J., M. C. Warren, and S. J. Clark, 1997, J. Phys.: Condens. Matter 9, 7861.

Allen, M. P., and D. J. Tildesley, 1987, Computer Simulation of Liquids (Clarendon Press, Oxford).

Althoff, J. D., D. Morgan, D. de Fontaine, M. Asta, S. M. Foiles, and D. D. Johnson, 1997, Phys. Rev. B 56, R5705.

Althoff, J. D., D. Morgan, D. de Fontaine, M. D. Asta, S. M. Foiles, and D. D. Johnson, 1998, Comput. Mater. Sci. 10, 411. Anthony, L., L. J. Nagel, J. K. Okamoto, and B. Fultz, 1994, Phys. Rev. Lett. 73, 3034.

Anthony, L., J. K. Okamoto, and B. Fultz, 1993, Phys. Rev. Lett. 70, 1128.

Ashcroft, N. W., and N. D. Mermin, 1976, Solid State Physics (Harcourt Brace College, New York).

Asta, M., R. McCormack, and D. de Fontaine, 1993, Phys. Rev. B 48, 748.

Bakker, H., 1982a, Philos. Mag. A 45, 213.

Bakker, H., 1982b, Phys. Status Solidi B 109, 211.

Bakker, H., and C. Tuijn, 1986, J. Phys. C 19, 5585.

Baroni, S., P. Giannozzi, and A. Testa, 1987, Phys. Rev. Lett. 58, 1861.

Becker, J. D., and J. M. Sanchez, 1993, Mater. Sci. Eng., A 170, 161.

Binder, K., and D. W. Heermann, 1988, Monte Carlo Simulation in Statistical Physics (Springer-Verlag, New York).

Bogdanoff, P. D., and B. Fultz, 1999, Philos. Mag. B 79, 753.

Bogdanoff, P. D., B. Fultz, and S. Rosenkranz, 1999, Phys. Rev. B 60, 3976.

Booth, C., and J. S. Rowlinson, 1955, Trans. Faraday Soc. 51, 463.
Born, M., and K. Huang, 1956, Dynamical Theory of Crystal Lattices (Oxford University Press, London).

Boyer, L. L., and M. J. Mehl, 1993, Ferroelectrics 150, 13. Ceder, G., 1991, Ph.D. thesis, University of California, Berkeley.

Ceder, G., 1993, Comput. Mater. Sci. 1, 144.

Ceder, G., M. Asta, W. C. Carter, M. Sluiter, M. E. Mann, M. Kraitchman, and D. de Fontaine, 1990, Phys. Rev. B 41, 8698. Chadi, D. J., and M. Cohen, 1973, Phys. Rev. B 8, 5747.

Cleri, F., and V. Rosato, 1993, Philos. Mag. Lett. 67, 369.

Colinet, C., et al., 1994, J. Phys.: Condens. Matter 6, L47.

Craievich, P. J., and J. M. Sanchez, 1997, Comput. Mater. Sci. 8, 92.

Craievich, P. J., J. Sanchez, R. E. Watson, and M. Weinert, 1997, Phys. Rev. B 55, 787.

Daw, M. S., and M. I. Baskes, 1984, Phys. Rev. B 29, 6443.

de Fontaine, D., 1994, Solid State Phys. 47, 33.

de Fontaine, D., J. Althoff, D. Morgan, M. Asta, S. Foiles, and D. J. A. Quong, 1998, in Phase Transformations and Systems Driven Far From Equilibrium, edited by E. Ma, M. Atzmon, P. Bellon, and R. Trivedi (Materials Research Society, Warrendale, PA), p. 175.

de Gironcoli, S., 1995, Phys. Rev. B 51, 6773.

Dick, B., and A. W. Overhausser, 1958, Phys. Rev. 112, 90.

Ducastelle, F., 1991, Order and Phase Stability in Alloys (Elsevier Science, New York).

Dyson, F. J., 1953, Phys. Rev. 92, 1331.

Fultz, B., L. Anthony, L. J. Nagel, J. K. Okamoto, R. M. Nicklow, and S. Spooner, 1995, Phys. Rev. B 52, 3315.

Fultz, B., L. Anthony, J. L. Robertson, R. M. Nicklow, S. Spooner, and M. Mostoller, 1995, Phys. Rev. B 52, 3280.

Fultz, B., T. A. Stephens, W. Sturhahn, T. S. Toellner, and E. E. Alp, 1998, Phys. Rev. Lett. 80, 3304.

Garbulsky, G. D., 1996, Ph.D. thesis, Massachusetts Institute of Technology, Cambridge, MA.

Garbulsky, G. D., and G. Ceder, 1994, Phys. Rev. B 49, 6327.

Garbulksy, G. D., and G. Ceder, 1995, Phys. Rev. B 51, 67.

Garbulsky, G. D., and G. Ceder, 1996, Phys. Rev. B 53, 8993.

Giannozzi, P., S. de Gironcoli, P. Pavone, and S. Baroni, 1991,

Phys. Rev. B 43, 7231.

Gonze, X., 1997, Phys. Rev. B 55, 10337.

Gonze, X., D. C. Allan, and M. P. Teter, 1992, Phys. Rev. Lett. 68, 3603 .

Gonze, X., and C. Lee, 1997, Phys. Rev. B 55, 10355.

Grimvall, G., 1986, Thermophysical Properties of Materials, Selected Topics in Solid State Physics, edited by E. P. Wohlfarth (North-Holland, Amsterdam).

Grimvall, G., and I. Ebbsjo, 1975, Phys. Scr. 12, 168.

Grimvall, G., and J. Rosen, 1983, Int. J. Thermophys. 4, 139.

Harrison, W. A., 1989, Electronic Structure and the Properties of Solids (Dover, New York).

Hass, K. C., L. C. Davis, and A. Zunger, 1990, Phys. Rev. B 42, 3757.

Imada, M., A. Fujimori, and Y. Tokura, 1998, Rev. Mod. Phys. 70, 1039.

Johnson, D. D., A. V. Smirnov, J. B. Staunton, F. J. Pinski, and W. A. Shelton, 2000, Phys. Rev. B 62, R11 917.

Kikuchi, R., 1951, Phys. Rev. 81, 988.

Kohan, A. F., P. D. Tepesch, G. Ceder, and C. Wolverton, 1998, Comput. Mater. Sci. 9, 389.

LeSar, R., R. Najafadabi, and D. J. Srolovitz, 1989, Phys. Rev. Lett. 63, 624. 
Maradudin, A. A., E. W. Montroll, and G. H. Weiss, 1971, Theory of Lattice Dynamics in the Harmonic Approximation, Second Edition (Academic Press, New York).

Matthew, J. A. D., R. E. Jones, and V. M. Dwyer, 1983, J. Phys. F: Met. Phys. 13, 581.

McCormack, R., and D. de Fontaine, 1996, Phys. Rev. B 54, 9746.

McQuarrie, D. A., 1973, Statistical Thermodynamics (University Science Books, Mill Valley, CA).

Miller, A., 1989, Phys. Rev. B 39, 3616.

Mohri, T., 1994, Prog. Theor. Phys. Suppl. 115, 147.

Mohri, T., S. Takizawa, and K. Terakura, 1993, J. Phys.: Condens. Matter 5, 1473.

Monkhorst, H. J., and J. Pack, 1976, Phys. Rev. B 13, 5188.

Moraitis, G., and F. Gautier, 1977, J. Phys. F: Met. Phys. 7, 1421.

Morgan, D., 1998, Ph.D. thesis, University of California, Berkeley.

Morgan, D., J. D. Althoff, and D. de Fontaine, 1998, J. Phase Equilib. 19, 559.

Morgan, D., A. van de Walle, G. Ceder, J. D. Althoff, and D. de Fontaine, 2000, Modell. Simul. Mater. Sci. Eng. 8, 1.

Moruzzi, V. L., J. F. Janak, and K. Schwarz, 1988, Phys. Rev. B 37, 790 .

Mukherjee, G. D., C. Bansal, and A. Chatterjee, 1996, Phys. Rev. Lett. 76, 1876.

Mukherjee, G. D., C. Bansal, and A. Chatterjee, 1998, Int. J. Mod. Phys. B 12, 2233.

Nagel, L. J., L. Anthony, and B. Fultz, 1995, Philos. Mag. Lett. 72, 421.

Nagel, L. J., L. Anthony, J. K. Okamoto, and B. Fultz, 1997, J. Phase Equilib. 18, 551.

Nagel, L. J., B. Fultz, and J. L. Robertson, 1997, Philos. Mag. B 75, 681 .

Nagel, L. J., B. Fultz, J. L. Robertson, and S. Spooner, 1996, Phys. Rev. B 55, 2903.

Najafabadi, R., H. Y. Wang, D. J. Srolovitz, and R. LeSar, 1991, Acta Metall. Mater. 39, 3071.

Nakamura, K., and T. Mohri, 1993, Modell. Simul. Mater. Sci. Eng. 1, 143.

Oh, C.-S., T. Mohri, and D. N. Lee, 1994, Mater. Trans., JIM 35, 445.

Ozolinsš, V., 1996, Ph.D. thesis, Royal Institute of Technology,

Stockholm, Sweden.

Ozolinšs, V., and M. Asta, 2001, Phys. Rev. B 86, 448.

Ozolinšs, V., C. Wolverton, and A. Zunger, 1998a, Phys. Rev. B 57, 6427.

Ozolinš̌, V., C. Wolverton, and A. Zunger, 1998b, Phys. Rev. B 57, 4816.

Ozolinšs, V., C. Wolverton, and A. Zunger, 1998c, Phys. Rev. B 58, R5897.

Persson, K., M. Ekman, and G. Grimvall, 1999, Phys. Rev. B 60, 9999.

Petry, W., A. Heiming, J. Trampenau, M. Alba, C. Herzig, H. R. Schober, and G. Vogl, 1991, Phys. Rev. B 43, 10933.

Pettifor, D. G., 1992, in Electron Theory in Alloy Design, edited by D. Pettifor and A. H. Cottrell (The Institute of Materials, London), p. 81.

Quong, A. A., and A. Y. Lui, 1997, Phys. Rev. B 56, 7767.
Quong, A. A., and B. Klein, 1992, Phys. Rev. B 46, 10734.

Ravelo, R., J. Aguilar, M. Baskes, J. Angelo, B. Fultz, and B. L. Holian, 1998, Phys. Rev. B 57, 862.

Rittner, J. D., S. M. Foiles, and D. N. Seidman, 1994, Phys. Rev. B 50, 12004.

Robertson, J. L., B. Fultz, and H. N. Frase, 1999, Phys. Rev. B 60, 9329.

Russell, T. P., T. E. Karis, Y. Gallot, and A. M. Mayes, 1994, Nature (London) 368, 729.

Sanchez, J. M., and J. D. Becker, 1994, Prog. Theor. Phys. Suppl. 115, 45.

Sanchez, J. M., F. Ducastelle, and D. Gratias, 1984, Physica A 128A, 334.

Sanchez, J. M., J. P. Stark, and V. L. Moruzzi, 1991, Phys. Rev. B 44, 5411.

Shaojun, L., D. Suqing, and M. Benkun, 1998, Phys. Rev. B 58, 9705.

Silverman, A., A. Zunger, A. Kalish, and J. Adler, 1995, Phys. Rev. B 51, 10795.

Sluiter, M. H., M. Weinert, and Y. Kawazoe, 1999, Phys. Rev. B 59, 4100.

Sutton, A. P., 1989, Philos. Mag. A 60, 147.

Tepesch, P. D., A. F. Kohan, G. D. Garbulsky, G. Ceder, C. Coley, H. T. Stokes, L. L. Boyer, M. J. Mehl, B. P. Burton, K. J. Cho, and J. Joannopoulos, 1996, J. Am. Ceram. Soc. 49, 2033.

Tseng, W. T., and J. P. Stark, 1994, Philos. Mag. B 70, 919.

Tuijn, C., and H. Bakker, 1989, Phys. Status Solidi B 155, 107. van de Walle, A., 1995, Master's thesis, University of Waterloo, Waterloo, Canada.

van der Ven, A., M. K. Aydinol, G. Ceder, G. Kresse, and J. Hafner, 1998, Phys. Rev. B 58, 2975.

van de Walle, A., and G. Ceder, 1999, Phys. Rev. B 59, 14992. van de Walle, A., and G. Ceder, 2000, Phys. Rev. B 61, 5972.

van de Walle, A., G. Ceder, and U. V. Waghmare, 1998, Phys. Rev. Lett. 80, 4911.

Voter, A. F., and S. P. Chen, 1988, in Characterization of Defects in Materials, edited by R. W. Siegel, J. R. Weertman, and R. Sinclair (Materials Research Society, Pittsburgh), p. 175.

Waegmaekers, A. A. H. J., and H. Bakker, 1984, in Phase Transformations in Solids, edited by T. Tsakalakos, Mater. Res. Soc. Symp. Proc. No. 21 (Materials Research Society, Pittsburgh, PA), p. 343.

Waghmare, U. V., 1996, Ph.D. thesis, Yale University, New Haven, CT.

Wei, S., and M. Y. Chou, 1992, Phys. Rev. Lett. 69, 2799.

Wojtowicz, P. J., and J. G. Kirkwood, 1960, J. Chem. Phys. 33, 1299.

Wolverton, C., and A. Zunger, 1995, Phys. Rev. B 52, 8813.

Wolverton, C., and V. Ozoliņš, 2001, Phys. Rev. Lett. 86, 5518.

Zhao, L., R. Najafabadi, and D. J. Srolovitz, 1993, Modell. Simul. Mater. Sci. Eng. 1, 539.

Zunger, A., 1994, in NATO Advanced Study Institute on Statics and Dynamics of Alloy Phase Transformation, edited by P. E. Turchi, and A. Gonis (Plenum Press, New York), Vol. 319, p. 361.

Zunger, A., S.-H. Wei, L. Ferreira, and J. E. Bernard, 1990, Phys. Rev. Lett. 65, 353. 